\title{
Green functions and the Dirichlet spectrum
}

\author{
G. Pacelli Bessa, Vicent Gimeno and Luquesio Jorge
}

\begin{abstract}
This article has results of four types. We show that the first eigenvalue $\lambda_{1}(\Omega)$ of the weighted Laplacian of a bounded domain with smooth boundary can be obtained by S. Sato's iteration scheme of the Green operator, taking the limit $\lambda_{1}(\Omega)=\lim _{k \rightarrow \infty}\left\|G^{k}(f)\right\|_{L^{2}} /\left\|G^{k+1}(f)\right\|_{L^{2}}$ for any $f \in L^{2}(\Omega, \mu), f>0$, (Theorems $2.1 \& 2.2$ ). Then, we study the $L^{1}(\Omega, \mu)$-moment spectrum of $\Omega$ in terms of iterates of the Green operator $G$, (Theorem 3.2), extendind the work of MacDonald-Meyers [29] to the weighted setting. As corollary, we obtain the first eigenvalue of a weighted bounded domain in terms of the $L^{1}(\Omega, \mu)$-moment spectrum, generalizing the work of Hurtado-Markvorsen-Palmer [25]. Finally, we study the radial spectrum $\sigma^{\mathrm{rad}}\left(B_{h}(o, r)\right)$ of rotationally invariant geodesic balls $B_{h}(o, r)$ of model manifolds. In Theorems $5.4 \& 5.6$, we prove an identity relating the radial eigenvalues of $\sigma^{\mathrm{rad}}\left(B_{h}(o, r)\right)$ to an isoperimetric quotient, i.e. $\sum 1 / \lambda_{i}^{\mathrm{rad}}=\int V(s) / S(s) d s, V(s)=\operatorname{vol}\left(B_{h}(o, s)\right)$ and $S(s)=\operatorname{vol}\left(\partial B_{h}(o, s)\right)$. We then consider a proper minimal surface $M \subset \mathbb{R}^{3}$ and the extrinsic ball $\Omega=M \cap B_{\mathbb{R}^{3}}(o, r)$. We obtain upper and lower estimates for the series $\sum \lambda_{i}^{-2}(\Omega)$ in terms of the volume $\operatorname{vol}(\Omega)$ and the radius $r$ of the extrinsic ball $\Omega$, (Theorem 6.1).
\end{abstract}

\section{Introduction}

sec:intro

A weighted manifold is a triple $\left(M, d s^{2}, \mu\right)$ consisting of a Riemannian manifold $\left(M, d s^{2}\right)$ and a measure $\mu$ with positive density function $\psi \in C^{\infty}(M), d \mu=\psi d \nu$, where $d \nu$ is the Riemannian density of $\left(M, d s^{2}\right)$. Let $\Omega \subset M$ be a relatively compact open subset with smooth boundary $\partial \Omega \neq \emptyset$ and consider the weighted Laplace operator $\triangle_{\mu}: C_{0}^{\infty}(\Omega) \rightarrow C_{0}^{\infty}(\Omega)$, acting on $C_{0}^{\infty}(\Omega)$, the space of smooth functions with compact support on $\Omega$, defined by

$$
\triangle_{\mu}=\frac{1}{\psi} \operatorname{div}(\psi \cdot \operatorname{grad}) \text {. }
$$

The weighted Laplace operator is densely defined and symmetric with respect to $L^{2}(\Omega, \mu)$-inner product, however, as in the classical case, it is not self-adjoint. We

Mathematics Subject Classification (2010): Primary 58C40, 35P15; Secondary 35J08.

Keywords: Dirichlet spectrum, radial spectrum, Green functions, rotationally invariant balls.. 
may consider the Sobolev spaces $W_{0}^{1}(\Omega, \mu)$ as the closure of $C_{0}^{\infty}(\Omega)$ with respect to the norm

$$
\|u\|_{W^{1}(\Omega, \mu)}^{2}:=\int_{\Omega} u^{2} d \mu+\int_{\Omega}|\operatorname{grad} u|^{2} d \mu
$$

and $W_{0}^{2}(\Omega, \mu)$, formed by those functions $u \in W_{0}^{1}(\Omega, \mu)$ whose weak Laplacian $\triangle_{\mu} u$ exists and belongs to $L^{2}(\Omega, \mu)$, i.e.

$$
W_{0}^{2}(\Omega, \mu)=\left\{u \in W_{0}^{1}(\Omega, \mu): \triangle_{\mu} u \in L^{2}(\Omega, \mu)\right\} .
$$

The operator $\mathcal{L}=-\left.\triangle_{\mu}\right|_{W_{0}^{2}(\Omega, \mu)}$ is a self-adjoint, non-negative definite elliptic operator and its spectrum, denoted by $\sigma(\Omega, \mu)$, is a discrete increasing sequence of non-negative real numbers $\left\{\lambda_{k}\right\}_{k=1}^{\infty} \subset[0, \infty)$, (counted according to multiplicity), with $\lim _{k \rightarrow \infty} \lambda_{k}=\infty$, (see [22, Thm.10.3]). The weighted Laplace operator $\triangle_{\mu}$ extends the classical Laplace operator $\triangle$, in the sense that, if the density function $\psi \equiv 1$ then $\triangle_{\mu}=\triangle$.

The weighted Green operator $G^{\Omega}: L^{2}(\Omega, \mu) \rightarrow L^{2}(\Omega, \mu)$, given by

$$
G^{\Omega}(f)(x)=\int_{0}^{\infty} \int_{\Omega} p_{t}^{\Omega}(x, y) f(y) d \mu(y) d t
$$

where $p_{t}^{\Omega}(x, y)$ is the heat kernel of the operator $\mathcal{L}=-\left.\triangle_{\mu}\right|_{W_{0}^{2}}(\Omega, \mu)$, is a bounded self-adjoint operator, inverse to $\mathcal{L}$, i.e. $G^{\Omega}=\mathcal{L}^{-1}$. For any $f \in L^{2}(\Omega, \mu)$ there is a unique solution $u=G^{\Omega}(f) \in W_{0}^{2}(\Omega, \mu)$ to the equation $-\triangle_{\mu} u=f$. In particular, applying $G^{\Omega}$ to the $i^{\text {th }}$-eigenvalue equation

$$
\triangle_{\mu} u_{i}+\lambda_{i}(\Omega) u_{i}=0
$$

we obtain the $i^{\text {th }}$-eigenvalue $\lambda_{i}(\Omega)=u_{i} / G^{\Omega}\left(u_{i}\right)$. The difficulty with this approach, in order to obtain the $i^{\text {th }}$-eigenvalue, is that one needs to know the Green operator and the $i^{\text {th }}$-eigenfunction beforehand. To overcome this inconvenience, in the main result $^{1}$ of Section 2, (Theorem 2.1), we apply a bootstrapping argument due to S. Sato [38] to show that we can obtain the first eigenvalue $\lambda_{1}(\Omega)$ as

$$
\lambda_{1}(\Omega)=\lim _{k \rightarrow \infty} \frac{\left\|G^{k}(f)\right\|_{L^{2}}}{\left\|G^{k+1}(f)\right\|_{L^{2}}}
$$

for any positive function $f \in L^{2}(\Omega, \mu)$, where $G^{k}=\overbrace{G \circ \cdots G}^{k-\text { times }}$. When $\Omega=B_{h}(o, r)$ is a rotationally invariant and $\triangle_{\mu}=\triangle$ then $G$ can be given explicitly, and by this result, we can give good numerical approximations for the first eigenvalue of $B_{h}(o, r)$, (Theorem 2.2).

In Section 3 we consider the following hierarchy Dirichlet problem on a bounded open subset with smooth boundary $\Omega \subset M$,

$$
\left\{\begin{aligned}
\phi_{0} & =1 \text { in } \Omega \\
\triangle_{\mu} \phi_{k}+k \phi_{k-1} & =0 \text { in } \Omega \\
\phi_{k} & =0 \text { on } \partial \Omega .
\end{aligned}\right.
$$

\footnotetext{
${ }^{1}$ For simplicity of notation, if no ambiguity arises, we will suppress the reference to $\Omega$ in the gadgets associated to $\Omega$. Thus, $G$ will be the Green operator of the operator $\mathcal{L}=-\left.\triangle_{\mu}\right|_{W_{0}^{2}(\Omega, \mu)}$.
} 
The set $\left\{\mathcal{A}_{k}\right\}_{k=1}^{\infty}, \mathcal{A}_{k}=\int_{\Omega} \phi_{k} d \mu$ is called the $L^{1}(\Omega, \mu)$-moment spectrum of $\Omega$. The $L^{1}(\Omega, \mu)$-moment spectrum is intertwined with the exit time and the Dirichlet spectrum of $\Omega$. Indeed, let us consider the hierarchy Dirichlet problem (1.1) for the Laplacian on a bounded open subset with smooth boundary $\Omega \subset M$. Suppose $X_{t}$ is a Brownian motion in $\Omega$ and $\tau=\inf \left\{t \geq 0: X_{t} \notin \Omega\right\}$ is the first exit time from $\Omega$. If $\phi_{k}$ is the solution of (1.1) then $\mathbb{E}^{x}\left[\tau^{k}\right]=\phi_{k}(x)$. The generator of the process $X_{t}$ associated to weighted Laplacian preserves the relationship between the exit time and the solutions of the hierarchy problem 1.1 as in the Laplacian case, (for an explanation and proof see [28]). The main result of this section is Theorem 3.2. There, we show that $\phi_{k}=k ! G^{k}(1)$, where $G$ is the $\triangle_{\mu}$-Green operator of $\Omega$. This formula was proven by MacDonald and Meyers [29, Formula 2.9, Prop. 2.2] in the non-weighted setting. We also have that $\lambda_{1}(\Omega)=\lim _{k \rightarrow \infty} k \mathcal{A}_{k-1} / \mathcal{A}_{k}$ extending the work of Hurtado-Markvorsen-Palmer [25], to general bounded domains, on the first eigenvalue of rotationally invariant balls $B_{h}(o, r)$.

In Section 4 we consider the eigenfunction $L^{2}$-expansion of the Green kernel of the weighted Laplacian. Let $\Omega$ be a relatively compact open subset of a Riemannian weighted manifold $M$ with Green function $g^{\Omega}: \Omega \times \Omega \rightarrow \mathbb{R}$. Let $\left\{u_{i}\right\}_{i=1}^{\infty}$ be an orthonormal basis of eigenfunctions of the Laplacian. An immediate corollary of Weyl's asymptotic formula $[21$, p. 7$]$ is that the necessary and sufficient condition for the the expansion of the Green function

$$
g(x, y)=\sum_{i=1}^{\infty} \frac{u_{i}(x) \cdot u_{i}(y)}{\lambda_{i}(\Omega)}
$$

to hold in $L^{2}$-sense is that $\operatorname{dim}(\Omega)=1,2,3$. The convergence of formal expansions of integral kernels such as the Green function is a classic problem in Mathematics. For instance, Mercer's Theorem states that, in dimension one, the convergence of (1.2) is absolute and uniform, (see [14, Chapter 3, Sec 4]). If $\Omega \subset \mathbb{R}^{n}, n=2,3$, this expansion is known to converge in $L^{2}$-sense, (see [14, Chapt. 5, Sec. 5]) and its proof can naturally adapted to domains in Riemannian manifolds, since it follows from Weyl's asymptotic formula. We formalize this observation as Theorem 4.1 , showing that $(1.2)$ holds in $L^{2}(\Omega, \mu)$ sense in dimension $n=1,2,3$ also for the Green kernel of weighted Laplacian. In higher dimension the negative result follows from bounds on the Green function, (see [15, Thm. 3.1.1]).

The spectrum of $\mathcal{L}=-\left.\triangle_{\mu}\right|_{W_{0}^{2}(\Omega, \mu)}$, sometimes called the spectrum of $\Omega$ and denoted by $\sigma(\Omega)$, is the set of all $\lambda \in[0, \infty)$ for which $\mathcal{L}-\lambda I$ is not injective or the inverse operator $(\mathcal{L}-\lambda I)^{-1}$ is unbounded, see [16]. The set of all $\lambda$ for which $(\mathcal{L}-\lambda I)$ is not injective is called the point spectrum and denoted by $\sigma_{p}(\Omega)$. The elements of $\sigma_{p}(\Omega)$ are the eigenvalues of $\mathcal{L}$. Each eigenvalue $\lambda \in \sigma_{p}(\Omega)$ defines an associated vector space $V_{\lambda}=\left\{u \in L^{2}(\Omega): \Delta u+\lambda u=0\right\}$. The set of all isolated eigenvalues of finite multiplicity, i.e., those $\lambda \in \sigma_{p}(\Omega)$ for which there exists $\epsilon>0$ such that $(\lambda-\epsilon, \lambda+\epsilon) \cap \sigma(\Omega)=\{\lambda\}$ and $\operatorname{dim}\left(V_{\lambda}\right)<\infty$ is called the discrete spectrum and it is denoted by $\sigma_{d}(\Omega)$. The complement of the discrete spectrum is the essential spectrum, $\sigma_{\text {ess }}(\Omega)=\sigma(M) \backslash \sigma_{d}(\Omega)$. When $\Omega$ is a bounded open set with smooth boundary $\partial \Omega$, (possibly empty), then the spectrum of $\mathcal{L}$ is discrete, 
i.e. a sequence of non-negative real numbers

$$
0 \leq \bar{\lambda}_{1}(\Omega)<\overline{\lambda_{2}}(\Omega)<\cdots \nearrow \infty
$$

where each associated eigenspace $V_{k}=\left\{\phi: \triangle \phi+\bar{\lambda}_{k} \phi=0\right\}$ is finite dimensional and $L^{2}(\Omega)=\oplus_{k=1}^{\infty} V_{k}$. Moreover, $\phi \in V_{k} \Rightarrow \phi \in C^{\infty}(\Omega) \cap C^{0}(\bar{\Omega})$ and $\phi \mid \partial \Omega=0$, ([11], [16] and [22, Thm.10.3]) $)^{2}$. The main geometric problem here is to describe the spectrum of a given open subset of a Riemannian manifold in terms of its geometry. To describe the spectrum of an open set in full generality is a difficult task (see [3], [4] and references therein). A more attainable problem is the study of the spectrum of rotationally invariant geodesic balls, i.e. balls of model manifolds $\mathbb{M}_{h}^{n}$ with center at the origin, (see Section 5 for the details). The spectrum $\sigma\left(B_{h}(o, r)\right)$ of a rotationally invariant geodesic ball $B_{h}(o, r)$ can be decomposed as a union of spectra $\sigma^{l}\left(B_{h}(o, r)\right)$ of a family of operators $L_{l}$ acting on smooth functions on $[0, r]$ indexed by the eigenvalues of the sphere $\nu_{l}=l(l+n-2), l=0,1, \ldots$, and we write, $\sigma\left(B_{h}(o, r)\right)=\cup_{l=0}^{\infty} \sigma^{l}\left(B_{h}(o, r)\right)$, (see [11, p. 41], [13, Chapters $\left.7 \& 8\right]$ ). Our first result concerns the spectrum $\sigma^{0}\left(B_{h}(o, r)\right)$. More precisely, we show in Theorem 5.4 that

$$
\sum_{\lambda \in \sigma^{0}\left(B_{h}(o, r)\right)} \lambda^{-1}=\int_{0}^{r} V(s) / S(s) d s,
$$

where $V(s)=\operatorname{vol}\left(B_{h}(o, s)\right)$ and $S(s)=\operatorname{vol}\left(\partial B_{h}(o, s)\right)$. When $B_{t}(o, r) \subset \mathbb{R}^{n}$ we obtain a similar result. Indeed, Theorem 5.6 states that

$$
\sum_{\lambda \in \sigma^{l}\left(B_{t}(o, r)\right)} \lambda^{-1}=c(n, l) \cdot \int_{0}^{r} V(s) / S(s) d s
$$

where $c(n, l)$ is a constant depending only on the dimension $n$ of the ambient space and $l$. To conclude the Section 5 , regarding the spectrum of rotationally invariant balls, we construct examples of 4-dimensional non-rotationally invariant geodesic balls $B_{h}(o, r)$ with the same spectrum $\sigma^{0}\left(B_{h}(o, r)\right)=\sigma^{0}\left(B_{h(t)=\sinh (t)}(o, r)\right)$ as the geodesic ball $\left.B_{\sinh (t)}(o, r)\right)$ of the hyperbolic space $\mathbb{H}^{4}(-1)$, (see Example 5.3).

In Section 6, we consider $m$-dimensional, $m=2,3$, proper isometric minimal immersions $\varphi: M \rightarrow \mathbb{R}^{n}$, with $\varphi(p)=o$ and extrinsic balls $\Omega_{r}$ of radius $r$, i.e. the connected component $\Omega_{r} \subset \varphi^{-1}\left(B_{h}(o, r)\right)$ containing $p$. The main result in this section, is the following eigenvalue estimate for $\Omega_{r}$,

$$
\frac{A_{m} r^{m}}{\operatorname{vol}\left(\Omega_{r}\right)} r^{4} \leq \sum_{\lambda \in \sigma\left(\Omega_{r}\right)} \lambda^{-2} \leq\left(\frac{\operatorname{vol}\left(\Omega_{r}\right)}{B_{m} r^{m}}\right)^{4 / m} r^{4},
$$

(see Theorem 6.1).

\footnotetext{
${ }^{2}$ Notation: in this article, the spectrum of $\Omega$ will be written either as a sequence of eigenvalues with repetition, according to their multiplicities, $\sigma(\Omega)=\left\{0 \leq \lambda_{1}(\Omega)<\lambda_{2}(\Omega) \leq \cdots\right\}$ or as a sequence of eigenvalues without repetition $\sigma(\Omega)=\left\{0 \leq \bar{\lambda}_{1}(\Omega)<\bar{\lambda}_{2}(\Omega)<\cdots\right\}$.
} 


\section{Weighted Green operator and the Dirichlet spectrum}

Let $(\Omega, \mu)$ be a weighted bounded open subset with smooth boundary $\partial \Omega \neq \emptyset$ of a Riemannian weighted manifold $\left(M, d s^{2}, \mu\right)$. The (weighted) Green operator $G^{\Omega}: L^{2}(\Omega, \mu) \rightarrow L^{2}(\Omega, \mu)$ is given by

$$
\begin{aligned}
G^{\Omega}(f)(x) & =\int_{0}^{\infty} \int_{\Omega} p_{t}^{\Omega}(x, y) f(y) d \mu(y) d t \\
& =\int_{\Omega} g^{\Omega}(x, y) f(y) d y
\end{aligned}
$$

where $p_{t}^{\Omega}(x, y)$ is the heat kernel of the operator $\mathcal{L}=-\left.\triangle_{\mu}\right|_{W_{0}^{2}(\Omega, \mu)}$ and

$$
g^{\Omega}(x, y)=\int_{0}^{\infty} p_{t}^{\Omega}(x, y) d t
$$

is the Green function of $\Omega$. The Green operator is a bounded self-adjoint operator in $L^{2}(\Omega, \mu)$ and it is the inverse of $\mathcal{L}$, i.e., $G^{\Omega}=\mathcal{L}^{-1}$. Thus for any $f \in L^{2}(\Omega, \mu)$ there is a unique solution $u=G^{\Omega}(f) \in W_{0}^{2}(\Omega, \mu)$ to the equation $-\triangle_{\mu} u=f$. If $f \in C_{0}^{\infty}(\Omega)$ then $G^{\Omega}(f) \in C^{\infty}(\Omega)$ see details in [22, Thm. 13.4]. Applying $G^{\Omega}$ to the equation

$$
\triangle_{\mu} u+\lambda_{i}(\Omega) u=0
$$

we obtain that the $i$-th eigenvalue $\lambda_{i}(\Omega)$ of $\Omega$ is given by $\lambda_{i}(\Omega)=u / G^{\Omega}(u)$. The difficulty to know precisely the $i^{\text {th }}$-eigenvalue applying the Green operator is that one needs to know an $i^{\text {th }}$ eigenfunction to start. For simplicity of notation, if no ambiguity arises, we will suppress the reference to $\Omega$ in the gadgets associated to $\Omega$. Thus, $g$ and $G$ will be, respectively, the Green function and the Green operator of the operator $\mathcal{L}=-\left.\triangle_{\mu}\right|_{W_{0}^{2}(\Omega, \mu)}$. Our main result in this section is that in order to obtain the first Dirichlet eigenvalue, assuming $\lambda_{1}(\Omega)>0$, one picks a positive function $f \in L^{2}(\Omega, \mu)$ and compute the limit

$$
\lambda_{1}=\lim _{k \rightarrow \infty} \frac{\left\|G^{k}(f)\right\|_{L^{2}}}{\left\|G^{k+1}(f)\right\|_{L^{2}}}
$$

where $\|u\|_{L^{2}}=\sqrt{\int_{\Omega}|u|^{2} d \mu}$ is the $L^{2}(\Omega, \mu)$-norm and $G^{k}=\overbrace{G \circ \cdots \circ G}^{k-\text { times }}$.

More generally, let $f \in L^{2}(\Omega, \mu)$ and let $\ell$ be the smallest positive integer such that

$$
\int_{\Omega} f(x) u_{i}(x) d \mu(x)=0
$$

for $i=1,2, \ldots, \ell-1$ and $\int_{\Omega} f(x) u_{\ell}(x) d \mu(x) \neq 0$, where $\left\{u_{\ell}\right\}$ is an orthonormal basis of $L^{2}(\Omega, \mu)$ formed by eigenfunctions $u_{\ell}$ with eigenvalues $\lambda_{\ell}$. Then the $\ell^{t h}-$ eigenvalue is given by

$$
\lambda_{\ell}=\lim _{k \rightarrow \infty} \frac{\left\|G^{k}(f)\right\|_{L^{2}}}{\left\|G^{k+1}(f)\right\|_{L^{2}}} .
$$


Main2 Theorem 2.1 Let $\left(\Omega, d s^{2}, \mu\right)$ be a weighted bounded open subset of a weighted $m$ manifold $\left(M, d s^{2}, \mu\right)$, with smooth boundary $\partial \Omega \neq \emptyset$. Let $G$ be the Green operator of $\mathcal{L}$ and let $\left\{u_{i}\right\}$ be an orthonormal basis of $L^{2}(\Omega, \mu)$ formed with eigenfunctions $u_{i}$ with eigenvalues $\lambda_{i}$. Then, for any $f \in L^{2}(\Omega), f \neq 0$,

$$
\lim _{k \rightarrow \infty} \frac{\left\|G^{k}(f)\right\|_{L^{2}}}{\left\|G^{k+1}(f)\right\|_{L^{2}}}=\lambda_{\ell}
$$

where $\ell$ is the first positive integer such that,

(2.4) $\int_{\Omega} f(x) u_{i}(x) d \mu(x)=0$ for $i=1,2, \ldots, \ell-1 \quad$ and $\int_{\Omega} f(x) u_{\ell}(x) d \mu(x) \neq 0$.

Moreover,

$$
\lim _{k \rightarrow \infty} \frac{G^{k}(f)}{\left\|G^{k}(f)\right\|_{L^{2}}}=\phi_{\ell} \in \operatorname{ker}\left(\triangle_{\mu}+\lambda_{\ell}\right) \text { in } L^{2}(\Omega, \mu)
$$

In particular, for any positive $f \in L^{2}(\Omega, \mu)$,

$$
\lim _{k \rightarrow \infty} \frac{\left\|G^{k}(f)\right\|_{L^{2}}}{\left\|G^{k+1}(f)\right\|_{L^{2}}}=\lambda_{1} \text { and } \lim _{k \rightarrow \infty} \frac{G^{k}(f)}{\left\|G^{k}(f)\right\|_{L^{2}}}=u_{1} \text { in } L^{2}(\Omega, \mu) .
$$

In addition, if $f \in L^{2}(\Omega, \mu)$ satisfying (2.4) and denoting by $f_{1}$ the function defined by

$$
f_{1}=f-\lambda_{l} G(f)
$$

then, if $f_{1} \neq 0$,

$$
\lim _{k \rightarrow \infty} \frac{\left\|G^{k}\left(f_{1}\right)\right\|_{L^{2}}}{\left\|G^{k+1}\left(f_{1}\right)\right\|_{L^{2}}}=\lambda_{n}
$$

with

$$
\lambda_{n}>\lambda_{l}
$$

And

$$
\lim _{k \rightarrow \infty} \frac{G^{k}\left(f_{1}\right)}{\left\|G^{k}\left(f_{1}\right)\right\|_{L^{2}}}=\phi_{n} \in \operatorname{ker}\left(\triangle_{\mu}+\lambda_{n}\right) .
$$

The effectiveness of Theorem 2.1 is shown when $\Omega$ is a rotationally invariant geodesic ball of a model manifold $\mathbb{M}_{h}^{m}$ and $\triangle_{\mu}=\triangle$, (see section 5 ). Indeed, let $C^{\mathrm{rad}}\left(B_{h}(o, r)\right)$ be the set of continuous radial functions $\phi: B_{h}(o, r) \rightarrow \mathbb{R}, \phi(t, \theta)=$ $\phi(t)$. Define the operator $T: C^{\mathrm{rad}}\left(B_{h}(o, r)\right) \rightarrow C^{\mathrm{rad}}\left(B_{h}(o, r)\right)$ by

$$
T(\phi)(t, \theta)=\int_{t}^{r} \frac{\int_{0}^{\sigma} h^{m-1}(s) \phi(s) d s}{h^{m-1}(\sigma)} d \sigma .
$$

The operator $T$ is the Green operator for $-L_{0}(f)=-\left(f^{\prime \prime}+(m-1) \frac{h^{\prime}}{h} f^{\prime}\right)=-\triangle_{\mu}(f)$ for the measure $d \mu=\omega_{m-1} h^{m-1} d \nu$. To see this, notice that $T$ can be seen as an operator $T: C^{0}([0, r]) \rightarrow C^{0}([0, r])$ and a straight forward calculations shows that $T=-L_{0}^{-1}$. Hence, Theorem 2.1 can be applied to obtain the following result. 
theo3 Theorem 2.2 Let $B_{h}(o, r) \subset \mathbb{M}_{h}^{m}$ be rotationally invariant geodesic ball of a model manifold with boundary $\partial B_{h}(o, r) \neq \emptyset$. For any $\phi \in C^{\mathrm{rad}}\left(B_{h}(o, r)\right), \phi \neq 0$, we have

$$
\frac{\left\|T^{k} \phi\right\|_{L^{2}}}{\left\|T^{k+1} \phi\right\|_{L^{2}}} \rightarrow \lambda_{l}^{\mathrm{rad}}\left(B_{h}(o, r)\right) \quad \text { and } \quad \frac{T^{k} \phi}{\left\|T^{k} \phi\right\|_{L^{2}}} \rightarrow \phi_{l} \text { in } L^{2}
$$

as $k \rightarrow \infty$. With $\phi_{l} \in \operatorname{ker}\left(\triangle+\lambda_{l}\right), \lambda_{l}=\lambda_{l}\left(B_{h}(o, r)\right)$ is $l^{\text {th }}$-radial eigenvalue, where $l$ is the first integer such that

$$
\int_{B_{h}(o, r)} \phi u_{i} d \nu=0 \text { for } i=1,2, \ldots, \ell-1 \text { and } \int_{B_{h}(o, r)} \phi u_{\ell} d \nu \neq 0
$$

where $\left\{u_{i}\right\}$ is an orthonormal basis of $L^{2}([0, r], \mu)$ eigenfunctions of $L_{0}$. Thus, in particular, for any positive $\phi \in C^{\mathrm{rad}}\left(B_{h}(o, r)\right)$ we have

$$
\frac{\left\|T^{k} \phi\right\|_{L^{2}}}{\left\|T^{k+1} \phi\right\|_{L^{2}}} \rightarrow \lambda_{1}\left(B_{h}(o, r)\right) \text { and } \frac{T^{k} \phi}{\left\|T^{k} \phi\right\|_{L^{2}}} \rightarrow \phi_{1} \text { in } L^{2},
$$

as $k \rightarrow \infty$.

To show how efficient Theorem 2.2 is, we let $B_{h}(o, r)$ be the geodesic ball centered at the origin $o$ and radius $r$ in rotationally symmetric manifold $\mathbb{M}_{h}^{n}$. Consider the following two maps:

$$
\begin{gathered}
\mathcal{T}^{k}: C^{\mathrm{rad}}\left(B_{h}(o, r)\right) \rightarrow \mathbb{R}, \quad \mathcal{T}^{k}(\phi):=\frac{\left\|T^{k} \phi\right\|_{L^{2}}}{\left\|T^{k+1} \phi\right\|_{L^{2}}} \\
\mathcal{T}^{\infty}: C^{\mathrm{rad}}\left(B_{h}(o, r)\right) \rightarrow \mathbb{R}, \quad \mathcal{T}^{\infty}(\phi):=\lim _{k \rightarrow \infty} \frac{\left\|T^{k} \phi\right\|_{L^{2}}}{\left\|T^{k+1} \phi\right\|_{L^{2}}},
\end{gathered}
$$

and the following family of functions arising from the $\phi_{0}=1$

$$
\left\{\begin{array}{l}
\phi_{0}=1 \\
\phi_{k}=\phi_{k-1}-\left(\mathcal{T}^{\infty}\left(\phi_{k-1}\right)\right) T \phi_{k-1}
\end{array}\right.
$$

Applying Theorem 2.2 we can obtain a subset of the radial spectrum, namely,

$$
\left\{\mathcal{T}^{\infty}\left(\phi_{k}\right)\right\}_{k=0}^{\infty} \subset \sigma^{\mathrm{rad}}\left(B_{h}(o, r)\right)
$$

If $h(t)=t$ then $\mathbb{M}_{t}^{2}=\mathbb{R}^{2}$, and the radial spectrum of the $B(o, 1)$ is given by $\sigma^{\mathrm{rad}}\left(B(o, 1)=\left\{j_{0, k}^{2}\right\}_{k=1}^{\infty}\right.$, where $j_{0, k}$ is the $k^{\text {th }}$-zero of the Bessel function $J_{0}$. The following table shows the $\mathcal{T}^{j}\left(\phi_{i}\right), j=1,2,3,9$ and $i=0,1,2$. The first radial eigenvalue $j_{0,1}^{2}$ has been estimated by Sato and other authors. The interesting thing is that $\mathcal{T}^{9}\left(\phi_{1}\right)$ agrees with $j_{0,2}^{2}$ to the $4^{\text {th }}$-decimal place.

\begin{tabular}{|c|c|c|c|}
\hline & $\phi_{0}=1$ & $\phi_{1}$ & $\phi_{2}$ \\
\hline $\mathcal{T}^{1}$ & 5.80381 & 31.8311 & 85.7823 \\
$\mathcal{T}^{2}$ & 5.78388 & 30.6656 & 77.4423 \\
$\mathcal{T}^{3}$ & 5.78321 & 30.5022 & 75.5737 \\
$\mathcal{T}^{9}$ & 5.78319 & 30.4713 & 74.8874 \\
\hline$\lambda$ & $j_{0,1}^{2} \approx 5.78319$ & $j_{0,2}^{2} \approx 30.4713$ & $j_{0,3}^{2} \approx 74.887$ \\
\hline
\end{tabular}


Remark 2.3 This bootstrapping argument using the Green operator in Theorem 2.1 was discovered, and applied to a particular kind of functions, by S. Sato [38] to obtain explicit estimates for the first eigenvalue of spherical caps of $\mathbb{S}^{2}(1)$. It was applied by Barroso-Bessa [5] to obtain estimates for the first eigenvalue of balls in rotationally symmetric manifolds.

2.0.1. Proof of Theorem 2.1. Let $\left(\Omega, d s^{2}, \mu\right)$ be a bounded weighted open subset, with smooth boundary $\partial \Omega \neq \emptyset$, of a Riemannian weighted $m$-manifold $\left(M, d s^{2}, \mu\right)$. The Green operator $G: L^{2}(\Omega, \mu) \rightarrow L^{2}(\Omega, \mu)$ is given by

$$
\begin{aligned}
G(f)(x) & =\int_{0}^{\infty} \int_{\Omega} p_{t}(x, y) f(y) d \mu(y) d t \\
& =\int_{\Omega} g(x, y) f(y) d y
\end{aligned}
$$

where $p_{t}(x, y)$ is the heat kernel of the operator $\mathcal{L}=-\left.\triangle_{\mu}\right|_{W_{0}^{2}(\Omega, \mu)}$ and $g(x, y)$ is the Green function of $\Omega$. Let $\left\{u_{1}, u_{2}, u_{3}, \ldots\right\}$ be a complete $L^{2}(\Omega, \mu)$-orthonormal basis of $L^{2}(\Omega, \mu)$ formed by eigenfunctions $u_{i}$ with eigenvalue $\lambda_{i}(\Omega) \in \sigma(\Omega)$. The proof Theorem 2.1 is divided in few simple propositions.

Proposition 2.4 If $\lambda_{1}(\Omega)>0$ then the Green operator $G$ satisfies

$$
G(f)(x)=\sum_{i=1}^{\infty} \frac{f^{i} u_{i}(x)}{\lambda_{i}(\Omega)} \text { for any } f \in L^{2}(\Omega, \mu),
$$

where $f^{i}=\int_{\Omega} f(x) u_{i}(x) d \mu(x)$. Moreover,

$$
G^{k}(f)=\sum_{i=1}^{\infty} \frac{f^{i} u_{i}(x)}{\lambda_{i}^{k}(\Omega)}
$$

where $G^{k}=\overbrace{G \circ \cdots \circ G}^{k-t i m e s}$.

Let $\left\{u_{i}\right\}_{i=1}^{\infty}$ be a complete orthonormal basis of $L^{2}(\Omega)$ formed by eigenfunctions. Then for any $f \in L^{2}(\Omega, \mu)$ we have

$$
f(x)=\sum_{i=1}^{\infty} f^{i} u_{i}(x)=U^{k}+f_{k},
$$

where $f_{k}(x)=\sum_{k+1}^{\infty} f^{i} u_{i}(x)$ and $U^{k}(x)=\sum_{i=1}^{k} f^{i} u_{i}(x)$. It is straight forward that $f_{k} \in L^{2}(\Omega, \mu)$ and therefore $U^{k}=f-f_{k} \in L^{2}(\Omega, \mu)$, because we have that $\sum_{i=k+1}^{\infty}\left(f^{i}\right)^{2}<\infty$ by Bessel's theorem. Therefore,

$$
\left\|f-\sum_{i=1}^{k} f^{i} u_{i}(x)\right\|_{L^{2}}=\left\|f-U^{k}\right\|_{L^{2}}=\left\|f_{k}\right\|_{L^{2}} \rightarrow 0 \text { as } k \rightarrow \infty .
$$


For any $k \in \mathbb{N}$,

eqGimeno2

$$
\begin{aligned}
G(f)(x) & =\int_{\Omega} g(x, y) f(y) d \mu(y) \\
& =\int_{\Omega} g(x, y) U^{k}(y) d \mu(y)+\int_{\Omega} g(x, y) f_{k}(y) d \mu(y) \\
& =\int_{\Omega} g(x, y) \sum_{i=1}^{k} f^{i} u_{i}(y) d \mu(y)+\int_{\Omega} g(x, y) f_{k}(y) d \mu(y) \\
& =\sum_{i=1}^{k} f^{i} \int_{\Omega} g(x, y) u_{i}(y) d \mu(y)+G\left(f_{k}\right)(x) \\
& =\sum_{i=1}^{k} \frac{f^{i} u_{i}(x)}{\lambda_{i}(\Omega)}+G\left(f_{k}\right)(x) .
\end{aligned}
$$

Therefore, from (2.20) and [22, Exercise 13.6 \& Definition 14.1] we have

$$
\left\|G(f)-\sum_{i=1}^{k} \frac{f^{i} u_{i}}{\lambda_{i}(\Omega)}\right\|_{L^{2}}=\left\|G\left(f_{k}\right)\right\|_{L^{2}} \leq \frac{\left\|f_{k}\right\|_{L^{2}}}{\lambda_{1}(\Omega)} \rightarrow 0 \text { as } k \rightarrow \infty .
$$

This implies that $G(f)=\sum_{i=1}^{\infty} \frac{f^{i} u_{i}}{\lambda_{1}(\Omega)}$ by Fischer-Riesz Theorem, (see [18], [37]) and it proves $(2.17)$. We used that $G\left(u_{i}\right)(x)=\int_{\Omega} g(x, y) u_{i}(y) d \mu(y)=u_{i}(x) / \lambda_{i}(\Omega)$.

We will prove (2.18) by induction. Assume that

$$
G^{k}(f)(x)=\sum_{i=1}^{\infty} \frac{f^{i} u_{i}(x)}{\lambda_{i}^{k}(\Omega)} \in L^{2}(\Omega) .
$$

Then, since $\left(G^{k}(f)\right)^{i}=\frac{f^{i}}{\lambda_{i}^{k}(\Omega)}$, we have

$$
\begin{aligned}
G^{k+1}(f)(x) & =G\left(G^{k}(f)\right)(x) \\
& =\sum_{i=1}^{\infty} \frac{\left(G^{k}(f)\right)^{i} u_{i}(x)}{\lambda_{i}(\Omega)} \\
& =\sum_{i=1}^{\infty} \frac{f^{i} u_{i}(x)}{\lambda_{i}^{k+1}(\Omega)} .
\end{aligned}
$$

propl1 Proposition 2.5 Let $\Omega \subset M$ be a bounded open set with smooth boundary $\partial \Omega$ such that $\lambda_{1}(\Omega)>0$. Then, for any $f \in L^{2}(\Omega)$,

$$
\left\|G^{k}(f)\right\|_{L^{2}}^{2}=\sum_{i=1}^{\infty} \frac{\left(f^{i}\right)^{2}}{\lambda_{i}^{2 k}(\Omega)}
$$


Moreover, if $f \neq 0$,

eq2. 24

eq2. 25

$$
\lim _{k \rightarrow \infty} \frac{\left\|G^{k}(f)\right\|_{L^{2}}}{\left\|G^{k+1}(f)\right\|_{L^{2}}}=\lambda_{l}(\Omega),
$$

$$
\lim _{k \rightarrow \infty} \frac{G^{k}(f)}{\left\|G^{k}(f)\right\|_{L^{2}}} \rightarrow \phi_{l} \in \operatorname{Ker}\left(\triangle_{\mu}+\lambda_{l}\right) \text { in } L^{2},
$$

where $l$ is the first integer such that,

$$
\int_{\Omega} f(y) u_{l}(y) d \mu(y) \neq 0 .
$$

Identity (2.23) follows from equation (2.18) and Parseval's identity.

To prove the identities $(2.24) \&(2.25)$ we let $f \in L^{2}(\Omega, \mu)$ be such that $\int_{\Omega} f(y) u_{i}(y) d \mu_{y}=0$ for $i=1, \ldots, l-1$, where $\left\{u_{1}, u_{2}, \ldots,\right\}$ is an orthonormal basis of eigenfunctions of $L^{2}(\Omega, \mu)$. Using (2.18) we have

$$
\begin{aligned}
\frac{\left\|G^{k}(f)\right\|_{L^{2}}^{2}}{\left\|G^{k+1}(f)\right\|_{L^{2}}^{2}} & =\frac{\sum_{i=l}^{\infty} \frac{\left(f^{i}\right)^{2}}{\lambda_{i}^{2 k}(\Omega)}}{\sum_{i=l}^{\infty} \frac{\left(f^{i}\right)^{2}}{\lambda_{i}^{2 k+2}(\Omega)}} \\
& =\lambda_{l}^{2}(\Omega) \frac{\sum_{i=l}^{\infty}\left(f^{i}\right)^{2}\left(\frac{\lambda_{l}(\Omega)}{\lambda_{i}(\Omega)}\right)^{2 k}}{\sum_{i=l}^{\infty}\left(f^{i}\right)^{2}\left(\frac{\lambda_{l}(\Omega)}{\lambda_{i}(\Omega)}\right)^{2 k+2}} \\
& =\lambda_{l}^{2}(\Omega) \frac{\sum_{i=l}^{l+m_{l}-1}\left(f^{i}\right)^{2}+\sum_{i=l+m_{l}}^{\infty}\left(f^{i}\right)^{2}\left(\frac{\lambda_{l}(\Omega)}{\lambda_{i}(\Omega)}\right)^{2 k}}{\sum_{i=l}^{l+m_{l}-1}\left(f^{i}\right)^{2}+\sum_{i=l+m_{l}}^{\infty}\left(f^{i}\right)^{2}\left(\frac{\lambda_{l}(\Omega)}{\lambda_{i}(\Omega)}\right)^{2 k+2}}
\end{aligned}
$$

where $m_{l}$ is the multiplicity of $\lambda_{l}$. From (2.27) we have that

$$
\lambda_{l}^{2}(\Omega) \frac{\sum_{i=l}^{l+m_{l}-1}\left(f^{i}\right)^{2}}{\sum_{i=l}^{l+m_{l}-1}\left(f^{i}\right)^{2}+\left(\frac{\lambda_{l}(\Omega)}{\lambda_{l+m_{l}}(\Omega)}\right)^{2 k+2} \sum_{i=l+m_{l}}^{\infty}\left(f^{i}\right)^{2}} \leq \frac{\left\|G^{k}(f)\right\|_{L^{2}}^{2}}{\left\|G^{k+1}(f)\right\|_{L^{2}}^{2}}
$$

and

$$
\frac{\left\|G^{k}(f)\right\|_{L^{2}}^{2}}{\left\|G^{k+1}(f)\right\|_{L^{2}}^{2}} \leq \lambda_{l}^{2}(\Omega) \frac{\sum_{i=l}^{l+m_{l}-1}\left(f^{i}\right)^{2}+\left(\frac{\lambda_{l}(\Omega)}{\lambda_{l+m_{l}}(\Omega)}\right)^{2 k+2} \sum_{i=l+m_{l}}^{\infty}\left(f^{i}\right)^{2}}{\sum_{i=l}^{l+m_{l}-1}\left(f^{i}\right)^{2}} .
$$


Since $\frac{\lambda_{l}(\Omega)}{\lambda_{l+m_{l}}(\Omega)}<1$, taking limits we have

$$
\lim _{k \rightarrow \infty} \frac{\left\|G^{k}(f)\right\|_{L^{2}}^{2}}{\left\|G^{k+1}(f)\right\|_{L^{2}}^{2}}=\lambda_{l}^{2}(\Omega) .
$$

This proves identity (2.3) of Theorem 2.1. To prove identity (2.5) we define

$$
\phi_{k}:=\frac{G^{k}(f)}{\left\|G^{k}(f)\right\|_{L_{2}}}
$$

for every $k \geq 1$. Since $\phi_{k} \in L^{2}(\Omega, \mu)$ we have $\phi_{k}(x)=\sum_{i=1}^{\infty} \phi_{k}^{i} u_{i}(x)$, where $\left\{u_{i}\right\}$ is an orthonormal basis of $L^{2}(\Omega, \mu)$. We are going to prove that $\phi_{k}$ converges in the $L^{2}$ sense to a function $\phi_{\infty}$ when $k \rightarrow \infty$ and that $\phi_{\infty} \in \operatorname{Ker}\left(\triangle_{l}+\lambda_{l}\right)$. By the identities (2.17) and (2.23) we have that

$$
\phi_{k}^{i}=\int_{\Omega} \frac{G^{k}(f)}{\left\|G^{k}(f)\right\|} u_{i} d \mu=\frac{f^{i}}{\lambda_{i}^{k}(\Omega)} \frac{1}{\sqrt{\sum_{j=l}^{\infty} \frac{\left(f^{j}\right)^{2}}{\lambda_{j}^{2 k}(\Omega)}}}
$$

$$
=\left(\frac{\lambda_{l}(\Omega)}{\lambda_{i}(\Omega)}\right)^{k} \frac{f^{i}}{\sqrt{\sum_{j=l}^{l+m_{l}-1}\left(f^{j}\right)^{2}+\sum_{j=l+m_{l}}^{\infty}\left(f^{j}\right)^{2}\left(\frac{\lambda_{l}(\Omega)}{\lambda_{j}(\Omega)}\right)^{2 k}}} .
$$

The first step is to prove that $\left\{\phi_{k}\right\}$ is a Cauchy sequence. Observe that for any $N \geq 0$,

$$
\begin{aligned}
&\left\|\phi_{k}-\phi_{k+N}\right\|_{L^{2}(\Omega, \mu)}^{2}= \sum_{i=1}^{\infty}\left(\phi_{k}^{i}-\phi_{k+N}^{i}\right)^{2} \\
&= \sum_{i=1}^{\infty}\left(\left(\phi_{k}^{i}\right)^{2}+\left(\phi_{k+N}^{i}\right)^{2}-2 \phi_{k}^{i} \phi_{k+N}^{i}\right) \\
&= \sum_{i=l}^{l+m_{l}-1}\left(\left(\phi_{k}^{i}\right)^{2}+\left(\phi_{k+N}^{i}\right)^{2}-2 \phi_{k}^{i} \phi_{k+N}^{i}\right) \\
&+\sum_{i=l+m_{l}}^{\infty}\left(\left(\phi_{k}^{i}\right)^{2}+\left(\phi_{k+N}^{i}\right)^{2}-2 \phi_{k}^{i} \phi_{k+N}^{i}\right) \\
& \leq 2-2 \frac{\sum_{i=l}^{l+m_{l}-1}\left(f^{i}\right)^{2}}{\sqrt{\sum_{j=l}^{l+m_{l}-1}\left(f^{j}\right)^{2}+\left(\frac{\lambda_{l}(\Omega)}{\lambda_{l+m_{l}}(\Omega)}\right)^{2 k}\|f\|_{2}} \sqrt{\sum_{j=l}^{l+m_{l}-1}\left(f^{j}\right)^{2}+\left(\frac{\lambda_{l}(\Omega)}{\lambda_{l+m_{l}}(\Omega)}\right)^{2 k+2 N}\|f\|_{2}}}
\end{aligned}
$$




$$
\begin{aligned}
\left.+\sum_{i=l+m_{l}}^{\infty}\left(\frac{\lambda_{l}(\Omega)}{\lambda_{i}(\Omega)}\right)^{2 k} \frac{\left(f^{i}\right)^{2}}{\sum_{j=l}^{l+m_{l}-1}\left(f^{j}\right)^{2}}+\left(\frac{\lambda_{l}(\Omega)}{\lambda_{i}(\Omega)}\right)^{2 k+2 N} \frac{\left(f^{i}\right)^{2}}{\sum_{j=l}^{l+m_{l}-1}\left(f^{j}\right)^{2}}\right) \\
\leq \frac{\sum_{i=l}^{l+m_{l}-1}\left(f^{i}\right)^{2}}{\sum_{j=l}^{l+m_{l}-1}\left(f^{j}\right)^{2}+\left(\frac{\lambda_{l}(\Omega)}{\lambda_{l+m_{l}}(\Omega)}\right)^{2 k}\|f\|_{L^{2}}}+2\left(\frac{\lambda_{l}(\Omega)}{\lambda_{l+m_{l}}(\Omega)}\right)^{2 k} \frac{\|f\|_{L^{2}}}{\sum_{j=l}^{l+m_{l}-1}\left(f^{j}\right)^{2}} .
\end{aligned}
$$

Since $\lambda_{l}(\Omega)<\lambda_{l+m_{l}}(\Omega)$ we have that $\left\|\phi_{k}-\phi_{k+N}\right\|^{2} \rightarrow 0$ when $k \rightarrow \infty$. Thus there exists $\phi_{\infty} \in L^{2}(\Omega, \mu)$ such that $\left\|\phi_{k}-\phi_{\infty}\right\|_{L_{2}} \rightarrow 0$ when $k \rightarrow \infty$. By equation (2.29) we can conclude that

$$
\left(\frac{\lambda_{l}(\Omega)}{\lambda_{i}(\Omega)}\right)^{2 k} \frac{\left(f^{i}\right)^{2}}{\sum_{j=l}^{l+m_{l}-1}\left(f^{j}\right)^{2}+\left(\frac{\lambda_{l}(\Omega)}{\lambda_{l+m_{l}}(\Omega)}\right)^{2 k}\|f\|_{L^{2}}} \leq\left|\phi_{k}^{i}\right|^{2} \leq\left(\frac{\lambda_{l}(\Omega)}{\lambda_{i}(\Omega)}\right)^{2 k} \frac{\left(f^{i}\right)^{2}}{\sum_{j=l}^{l+m_{l}-1}\left(f^{j}\right)^{2}}
$$

taking into account that $f^{i}=0$, for $i<l$, and $\lambda_{l}(\Omega)<\lambda_{i}(\Omega)$ for $i \geq l+m_{l}$, we have that

$$
\begin{cases}\lim _{k \rightarrow \infty}\left|\phi_{k}^{i}\right|^{2}=0 & \text { if } \quad i<l \\ \lim _{k \rightarrow \infty}\left|\phi_{k}^{i}\right|^{2}=\frac{\left(f^{i}\right)^{2}}{\sum_{j=l}^{l+m_{l}-1}\left(f^{j}\right)^{2}} & \text { if } \quad l \leq i \leq l+m_{l}-1 \\ \lim _{k \rightarrow \infty}\left|\phi_{k}^{i}\right|^{2}=0, & \text { if } \quad i \geq l+m_{l}\end{cases}
$$

This shows that $\phi_{\infty}=\lim _{k \rightarrow \infty} \frac{G^{k}(f)}{\left\|G^{k}(f)\right\|_{L^{2}}} \in \operatorname{Ker}\left(\triangle_{l}+\lambda_{l}\right)=V_{l} \oplus \cdots \oplus V_{l+m_{l}-1}$ and proves identity (2.5) of Theorem 2.1.

cor17 Corollary 2.6 Under the assumptions of the above proposition and letting $f_{1}$ be the function

$$
f_{1}:=f-\lambda_{l}(\Omega) G(f),
$$

then, if $f_{1} \neq 0$, we have

$$
\begin{aligned}
& \lim _{k \rightarrow \infty} \frac{\left\|G^{k}\left(f_{1}\right)\right\|_{L^{2}}}{\left\|G^{k+1}\left(f_{1}\right)\right\|_{L^{2}}}=\lambda_{n}(\Omega) \\
& \lim _{k \rightarrow \infty} \frac{G^{k}\left(f_{1}\right)}{\left\|G^{k}\left(f_{1}\right)\right\|_{L^{2}}} \rightarrow \phi_{n} \in \operatorname{Ker}\left(\triangle_{\mu}+\lambda_{n}\right) \text { in } L^{2}
\end{aligned}
$$

with,

$$
\lambda_{n}(\Omega)>\lambda_{l}(\Omega) .
$$


We only have to apply the above proposition taking into account that by equality (2.17)

$$
\begin{aligned}
f-\lambda_{l}(\Omega) G(f) & =\sum_{i=l}^{\infty} f^{i} u_{i}-\sum_{i=l}^{\infty} f^{i} u_{i} \frac{\lambda_{l}(\Omega)}{\lambda_{i}(\Omega)} \\
& =\sum_{i=l+1}^{\infty} f^{i} u_{i}\left(1-\frac{\lambda_{l}(\Omega)}{\lambda_{i}(\Omega)}\right) .
\end{aligned}
$$

Observe that since $u_{1}$ does not change its sign in $\Omega$, we have that

$$
\int_{\Omega} f(x) u_{1}(x) d \mu(x) \neq 0
$$

for any positive or negative function $f$. Hence, using Proposition 2.5, we have

cor18 Corollary 2.7 Let $\Omega \subset M$ be a bounded open set with smooth boundary $\partial \Omega \neq \emptyset$. Then, for any positive (or negative) $f \in L^{2}(\Omega, \mu)$,

$$
\begin{aligned}
\lim _{k \rightarrow \infty} \frac{\left\|G^{k}(f)\right\|_{L^{2}}}{\left\|G^{k+1}(f)\right\|_{L^{2}}} & =\lambda_{1}(\Omega), \\
\lim _{k \rightarrow \infty} \frac{G^{k}(f)}{\left\|G^{k}(f)\right\|_{L^{2}}} & =\phi_{1} \in \operatorname{Ker}\left(\triangle_{\mu}+\lambda_{1}\right) \text { in } L^{2} .
\end{aligned}
$$

\section{3. $L^{1}(\Omega, \mu)$-moment spectrum}

Let $\left(\Omega, d s^{2}, \mu\right)$ be a bounded open subset of a weighted Riemannian manifold with smooth boundary $\partial \Omega \neq \emptyset$. Let $\phi_{k}: \Omega \rightarrow \mathbb{R}, k=0,1, \ldots$ be a sequence of functions defined inductively as the solutions to the following hierarchy of boundary value problems on $\Omega$. Let $\phi_{0}=1$ in $\Omega$ and for $k \geq 1$

$$
\left\{\begin{aligned}
\triangle_{\mu} \phi_{k}+k \phi_{k-1} & =0 \text { in } \Omega \\
\phi_{k} & =0 \text { on } \partial \Omega .
\end{aligned}\right.
$$

The solution $\phi_{1}(x)$ is the mean time for the first exit of $\Omega$ of a Brownian motion $t \rightarrow X_{t}$ in $\Omega$, with $X_{0}=x$.

Definition 3.1 The $L^{1}(\Omega, \mu)$-moment spectrum of $\Omega$ is the set $\left\{\mathcal{A}_{k}(\Omega)\right\}_{k=1}^{\infty}$ of the $L^{1}(\Omega, \mu)$-norms of $\phi_{k}$,

$$
\mathcal{A}_{k}(\Omega)=\int_{\Omega} \phi_{k} d \mu .
$$

The number $\mathcal{A}_{1}(\Omega)$ is called the torsional rigidity of $\Omega$ because, when $\Omega \subset \mathbb{R}^{2}$, $\mathcal{A}_{1}(\Omega)$ is the torque required for a unit angle of twist per unit length when twisting an elastic beam of uniform cross section $\Omega$, (see [25], [32] and [30]).

Observe that if $\Omega=B_{h}(o, r)$ is a rotationally invariant geodesic ball then the solutions $\phi_{k}(x)=\phi_{k}(|x|)$ of (3.1) are radial functions since $\phi_{1}$ is radial. In [25], 
A. Hurtado, S. Markvorsen and V. Palmer, showed that the first eigenvalue of a rotationally invariant ball can be given in terms of the moment spectrum. They proved that

$$
\lambda_{1}\left(B_{h}(o, r)\right)=\lim _{k \rightarrow \infty} \frac{k \phi_{k-1}(0)}{\phi_{k}(0)}=\lim _{k \rightarrow \infty} \frac{k \mathcal{A}_{k-1}\left(B_{h}(o, r)\right)}{\mathcal{A}_{k}\left(B_{h}(o, r)\right)}
$$

and $\phi_{\infty}(r)=\lim _{k \rightarrow \infty} \phi_{k}(r) / \phi_{k}(0)$ is a radial first eigenfunction. In this direction, it should be remarked that for rotationally invariant geodesic balls $B_{h}(o, r)$, BessaMontenegro (see [8]), proved that

$$
\lambda_{1}\left(B_{h}(o, r)\right) \geq \inf _{0 \leq t \leq r} \frac{1}{4}\left[\frac{S(t)}{V(t)}\right]^{2}
$$

In our next result we solve explicitly the problem (3.1) in terms of the Green operator $G$ for the weighted Laplacian $\triangle_{\mu}$. It links the moment spectrum with the Dirichlet spectrum of domains and in case of rotationally invariant geodesic balls and it recovers Hurtado-Markvorsen-Palmer's result.

thm4.2 Theorem 3.2 Let $\left(\Omega, d s^{2}, \mu\right)$ be a bounded open subset of a Riemannian weighted manifold $\left(M, d s^{2}, \mu\right)$ with smooth boundary $\partial \Omega \neq \emptyset$. Let $\phi_{k}$ be the sequence of functions given by the boundary problem (3.1) and $G$ the $\triangle_{\mu}$-Green operator of $\Omega$. Then

$$
\phi_{k}=k ! G^{k}(1) .
$$

Hence the exit moment spectrum is related to the Dirichlet spectrum as follows

eqphi_k2

$$
\begin{aligned}
\phi_{k}(x) & =k ! \sum_{i=1}^{\infty} \frac{a_{i} u_{i}(x)}{\lambda_{i}^{k}} \\
\mathcal{A}_{k} & =k ! \sum_{i=1}^{\infty} \frac{a_{i}^{2}}{\lambda_{i}^{k}}
\end{aligned}
$$

where $a_{i}=\int_{\Omega} u_{i}(x) d \mu(x)$ and $\left\{u_{i}\right\}$ is an orthonormal basis of $L^{2}(\Omega, \mu)$ formed by eigenfunctions of $\mathcal{L}=-\left.\triangle_{\mu}\right|_{W_{0}^{2}(\Omega, \mu)}$.

cor4.1 Corollary 3.3 Let $\left(\Omega, d s^{2}, \mu\right)$ be a bounded open subset of a Riemannian weighted manifold manifold $\left(M, d s^{2}, \mu\right)$ with smooth boundary $\partial \Omega \neq \emptyset$. Then,

$$
\lambda_{1}=\lim _{k \rightarrow \infty} \frac{k \mathcal{A}_{k-1}}{\mathcal{A}_{k}} \quad \text { and } \lambda_{2} \leq \lim _{k \rightarrow \infty} k \frac{(k-1) \mathcal{A}_{k-2}-\lambda_{1} \mathcal{A}_{k-1}}{k \mathcal{A}_{k-1}-\lambda_{1} \mathcal{A}_{k}} .
$$

Hence,

$$
\frac{\lambda_{2}}{\lambda_{1}} \leq \lim _{k \rightarrow \infty} \frac{\frac{(k-1) \mathcal{A}_{k-2}}{\mathcal{A}_{k-1}}-\lambda_{1}}{\frac{k \mathcal{A}_{k-1}}{\mathcal{A}_{k}}-\lambda_{1}}<\infty .
$$


The proof of the Corollary is as follows: Since we know (by Theorem 3.2) that $\mathcal{A}_{k}=k ! \sum_{i=1}^{\infty} \frac{a_{i}^{2}}{\lambda_{i}^{k}}$ then

$$
\begin{aligned}
\frac{k \mathcal{A}_{k-1}}{\mathcal{A}_{k}} & =\frac{\sum_{i=1}^{\infty} \frac{a_{i}^{2}}{\lambda_{i}^{k-1}}}{\sum_{i=1}^{\infty} \frac{a_{i}^{2}}{\lambda_{i}^{k}}} \\
& =\frac{\frac{a_{1}^{2}}{\lambda_{1}^{k-1}}+\sum_{i=2}^{\infty} \frac{a_{i}^{2}}{\lambda_{i}^{k-1}}}{\frac{a_{1}^{2}}{\lambda_{1}^{k}}+\sum_{i=2}^{\infty} \frac{a_{i}^{2}}{\lambda_{i}^{k}}} \\
& =\frac{a_{1}^{2} \lambda_{1}+\lambda_{1} \sum_{i=2}^{\infty} a_{i}^{2}\left(\frac{\lambda_{1}}{\lambda_{i}}\right)^{k-1}}{a_{1}^{2}+\sum_{i=2}^{\infty} a_{i}^{2}\left(\frac{\lambda_{1}}{\lambda_{i}}\right)^{k}}
\end{aligned}
$$

Now observe that, since $\lambda_{i}>\lambda_{1}$ for $i>1$,

$$
\begin{aligned}
0 \leq \sum_{i=2}^{\infty} a_{i}^{2}\left(\frac{\lambda_{1}}{\lambda_{i}}\right)^{k} & \leq \sum_{i=2}^{\infty} a_{i}^{2}\left(\frac{\lambda_{1}}{\lambda_{i}}\right)^{k-1}=\sum_{i=2}^{\infty} a_{i}^{2}\left(\frac{\lambda_{1}}{\lambda_{i}}\right)^{k-2}\left(\frac{\lambda_{1}}{\lambda_{i}}\right) \\
& \leq \sum_{i=2}^{\infty} a_{i}^{2}\left(\frac{\lambda_{1}}{\lambda_{2}}\right)^{k-2}\left(\frac{\lambda_{1}}{\lambda_{i}}\right)=\lambda_{1}\left(\frac{\lambda_{1}}{\lambda_{2}}\right)^{k-2} \mathcal{A}_{1}
\end{aligned}
$$

Hence, letting $k$ tend to infinity we conclude that

$$
\lim _{k \rightarrow \infty} \sum_{i=2}^{\infty} a_{i}^{2}\left(\frac{\lambda_{1}}{\lambda_{i}}\right)^{k-1}=\lim _{k \rightarrow \infty} \sum_{i=2}^{\infty} a_{i}^{2}\left(\frac{\lambda_{1}}{\lambda_{i}}\right)^{k}=0 .
$$

Therefore taking limits in equality (3.5) taking into account that

$$
\lim _{k \rightarrow \infty}\left(a_{1}^{2}+\sum_{i=2}^{\infty} a_{i}^{2}\left(\frac{\lambda_{1}}{\lambda_{i}}\right)^{k}\right)=a_{1}^{2} \neq 0,
$$

and $\lim _{k \rightarrow \infty}\left(a_{1}^{2} \lambda_{1}+\lambda_{1} \sum_{i=2}^{\infty} a_{i}^{2}\left(\frac{\lambda_{1}}{\lambda_{i}}\right)^{k-1}\right)=a_{1}^{2} \lambda_{1}$

$$
\lim _{k \rightarrow \infty} \frac{k \mathcal{A}_{k-1}}{\mathcal{A}_{k}}=\frac{a_{1}^{2} \lambda_{1}}{a_{1}^{2}}=\lambda_{1}
$$

Likewise,

$$
k \frac{(k-1) \mathcal{A}_{k-2}-\lambda_{1} \mathcal{A}_{k-1}}{k \mathcal{A}_{k-1}-\lambda_{1} \mathcal{A}_{k}}=\frac{\sum_{i=2}^{\infty} \frac{a_{i}^{2}}{\lambda_{i}^{k-2}}-\lambda_{1} \sum_{i=2}^{\infty} \frac{a_{i}^{2}}{\lambda_{i}^{k-1}}}{\sum_{i=2}^{\infty} \frac{a_{i}^{2}}{\lambda_{i}^{k-1}}-\lambda_{1} \sum_{i=2}^{\infty} \frac{a_{i}^{2}}{\lambda_{i}^{k}}}
$$

Let us denote by $\alpha$ the first integer such that $\alpha>1$ and $a_{\alpha}^{2} \neq 0$. Then

$$
\begin{aligned}
k \frac{(k-1) \mathcal{A}_{k-2}-\lambda_{1} \mathcal{A}_{k-1}}{k \mathcal{A}_{k-1}-\lambda_{1} \mathcal{A}_{k}} & =\frac{\sum_{i=\alpha}^{\infty} \frac{a_{i}^{2}}{\lambda_{i}^{k-2}}-\lambda_{1} \sum_{i=\alpha}^{\infty} \frac{a_{i}^{2}}{\lambda_{i}^{k-1}}}{\sum_{i=\alpha}^{\infty} \frac{a_{i}^{2}}{\lambda_{i}^{k-1}}-\lambda_{1} \sum_{i=\alpha}^{\infty} \frac{a_{i}^{2}}{\lambda_{i}^{k}}} \\
=I &
\end{aligned}
$$


We rewrite $I$ as

$$
I=\frac{a_{\alpha}^{2}\left(\lambda_{\alpha}-\lambda_{1}\right)+\lambda_{\alpha} \sum_{i=\alpha+1}^{\infty} a_{i}^{2}\left(\frac{\lambda_{\alpha}}{\lambda_{i}}\right)^{k-2}-\lambda_{1} \sum_{i=\alpha+1}^{\infty} a_{i}^{2}\left(\frac{\lambda_{\alpha}}{\lambda_{i}}\right)^{k-1}}{a_{\alpha}^{2}\left(1-\frac{\lambda_{1}}{\lambda_{\alpha}}\right)+\sum_{i=\alpha+1}^{\infty} a_{i}^{2}\left(\frac{\lambda_{\alpha}}{\lambda_{i}}\right)^{k-1}-\frac{\lambda_{1}}{\lambda_{\alpha}} \sum_{i=\alpha+1}^{\infty} a_{i}^{2}\left(\frac{\lambda_{\alpha}}{\lambda_{i}}\right)^{k}}
$$

Suppose that the eigenvalue $\lambda_{\alpha}$ has multiplicity $m_{\alpha}$. Then

$$
\sum_{i=\alpha+1}^{\infty} a_{i}^{2}\left(\frac{\lambda_{\alpha}}{\lambda_{i}}\right)^{k}=\sum_{i=\alpha}^{\infty} a_{i}^{2}\left(\frac{\lambda_{\alpha}}{\lambda_{i}}\right)^{k}-a_{\alpha}^{2}=\sum_{i=\alpha}^{\alpha+m_{\alpha}-1} a_{i}^{2}-a_{\alpha}^{2}+\sum_{i=\alpha+m_{\alpha}}^{\infty} a_{i}^{2}\left(\frac{\lambda_{\alpha}}{\lambda_{i}}\right)^{k}
$$

Using a completely similar argument as used before we can prove that

$$
\lim _{k \rightarrow \infty} \sum_{i=\alpha+m_{\alpha}}^{\infty} a_{i}^{2}\left(\frac{\lambda_{\alpha}}{\lambda_{i}}\right)^{k}=0
$$

Then

$$
\lim _{k \rightarrow \infty} \sum_{i=\alpha+1}^{\infty} a_{i}^{2}\left(\frac{\lambda_{\alpha}}{\lambda_{i}}\right)^{k}=\sum_{i=\alpha}^{\alpha+m_{\alpha}-1} a_{i}^{2}-a_{\alpha}^{2}
$$

Similarly,

$$
\lim _{k \rightarrow \infty} \sum_{i=\alpha+1}^{\infty} a_{i}^{2}\left(\frac{\lambda_{\alpha}}{\lambda_{i}}\right)^{k-2}=\lim _{k \rightarrow \infty} \sum_{i=\alpha+1}^{\infty} a_{i}^{2}\left(\frac{\lambda_{\alpha}}{\lambda_{i}}\right)^{k-1}=\sum_{i=\alpha}^{\alpha+m_{\alpha}-1} a_{i}^{2}-a_{\alpha}^{2}
$$

Hence taking into account that

$$
\begin{aligned}
& \lim _{k \rightarrow \infty} a_{\alpha}^{2}\left(\lambda_{\alpha}-\lambda_{1}\right)+\lambda_{\alpha} \sum_{i=\alpha+1}^{\infty} a_{i}^{2}\left(\frac{\lambda_{\alpha}}{\lambda_{i}}\right)^{k-2}-\lambda_{1} \sum_{i=\alpha+1}^{\infty} a_{i}^{2}\left(\frac{\lambda_{\alpha}}{\lambda_{i}}\right)^{k-1} \\
& =a_{\alpha}^{2}\left(\lambda_{\alpha}-\lambda_{1}\right)+\lambda_{\alpha}\left(\sum_{i=\alpha}^{\alpha+m_{\alpha}-1} a_{i}^{2}-a_{\alpha}^{2}\right)-\lambda_{1}\left(\sum_{i=\alpha}^{\alpha+m_{\alpha}-1} a_{i}^{2}-a_{\alpha}^{2}\right) \\
& =\left(\lambda_{\alpha}-\lambda_{1}\right) \sum_{i=\alpha}^{\alpha+m_{\alpha}-1} a_{i}^{2}
\end{aligned}
$$

and

$$
\begin{aligned}
& \lim _{k \rightarrow \infty}\left(a_{\alpha}^{2}\left(1-\frac{\lambda_{1}}{\lambda_{\alpha}}\right)+\sum_{i=\alpha+1}^{\infty} a_{i}^{2}\left(\frac{\lambda_{\alpha}}{\lambda_{i}}\right)^{k-1}-\frac{\lambda_{1}}{\lambda_{\alpha}} \sum_{i=\alpha+1}^{\infty} a_{i}^{2}\left(\frac{\lambda_{\alpha}}{\lambda_{i}}\right)^{k}\right)= \\
& =a_{\alpha}^{2}\left(1-\frac{\lambda_{1}}{\lambda_{\alpha}}\right)+\left(\sum_{i=\alpha}^{\alpha+m_{\alpha}-1} a_{i}^{2}-a_{\alpha}^{2}\right)-\frac{\lambda_{1}}{\lambda_{\alpha}}\left(\sum_{i=\alpha}^{\alpha+m_{\alpha}-1} a_{i}^{2}-a_{\alpha}^{2}\right) \\
& =\left(1-\frac{\lambda_{1}}{\lambda_{\alpha}}\right) \sum_{i=\alpha}^{\alpha+m_{\alpha}-1} a_{i}^{2} \neq 0
\end{aligned}
$$


we can take limits in equation (3.8)

$$
\lim _{k \rightarrow \infty} k \frac{(k-1) \mathcal{A}_{k-2}-\lambda_{1} \mathcal{A}_{k-1}}{k \mathcal{A}_{k-1}-\lambda_{1} \mathcal{A}_{k}}=\frac{\left(\lambda_{\alpha}-\lambda_{1}\right) \sum_{i=\alpha}^{\alpha+m_{\alpha}-1} a_{i}^{2}}{\left(1-\frac{\lambda_{1}}{\lambda_{\alpha}}\right) \sum_{i=\alpha}^{\alpha+m_{\alpha}-1} a_{i}^{2}}=\lambda_{\alpha} \geq \lambda_{2}
$$

Finally, since $\lambda_{1} \neq 0$,

$$
\begin{aligned}
\frac{\lambda_{2}}{\lambda_{1}} \leq \frac{\lambda_{\alpha}}{\lambda_{1}} & =\frac{\lim _{k \rightarrow \infty} k \frac{(k-1) \mathcal{A}_{k-2}-\lambda_{1} \mathcal{A}_{k-1}}{k \mathcal{A}_{k-1}-\lambda_{1} \mathcal{A}_{k}}}{\lim _{k \rightarrow \infty} \frac{k \mathcal{A}_{k-1}}{\mathcal{A}_{k}}} \\
& =\lim _{k \rightarrow \infty} \frac{\frac{(k-1) \mathcal{A}_{k-2}-\lambda_{1} \mathcal{A}_{k-1}}{k \mathcal{A}_{k-1}-\lambda_{1} \mathcal{A}_{k}}}{\frac{\mathcal{A}_{k-1}}{\mathcal{A}_{k}}} \\
& =\lim _{k \rightarrow \infty} \frac{\frac{(k-1) \mathcal{A}_{k-2}}{\mathcal{A}_{k-1}}-\lambda_{1}}{\frac{k \mathcal{A}_{k-1}}{\mathcal{A}_{k}}-\lambda_{1}} .
\end{aligned}
$$

In the second part of this proof we have assumed that there existed an $\alpha>1$ with $a_{\alpha}=\int_{\Omega} u_{\alpha}(x) d \mu(x) \neq 0$. But this is true because $\left\{u_{i}\right\}_{i=1}^{\infty}$ is complete orthonormal basis of $L^{2}(\Omega, \mu)$. Then since $1 \in L^{2}(\Omega, \mu)$,

$$
1=\sum_{i=1}^{\infty} u_{i}(x) \int_{\Omega} u_{i}(y) d \mu(y)=\sum_{i=1}^{\infty} a_{i} u_{i}(x)
$$

Suppose by the contrary that $a_{i}=0$ for any $i>1$ then this should imply that

$$
1=a_{1} u_{1}(x) \Rightarrow u_{1}(x)=\frac{1}{a_{1}}
$$

but this is a contradiction because $u_{1 \mid \partial \Omega}=0$ and $u_{1} \in C_{2}(\Omega) \cap C_{0}(\bar{\Omega})$.

3.0.1. Proof of Theorem 3.2 . Let $\Omega$ be an open relatively compact domain with smooth boundary $\partial \Omega \neq \emptyset$ of a Riemannian manifold $M$. Let us consider the hierarchy Dirichlet problem (1.1) Theorem 3.2 states that this problem admits a unique family of solutions $\left\{\phi_{k}\right\}_{k=1}^{\infty}$, given by

$$
\phi_{k}(x)=k ! G^{k}(1)(x) .
$$

In order to show the uniqueness, note that first of all that $\phi_{1}$ is unique. Otherwise we would have two different functions $\phi_{1}^{1}$ and $\phi_{1}^{2}$ such that

$$
\begin{aligned}
& \triangle \phi_{1}^{1}=\triangle \phi_{1}^{2}=-1 \\
& \left.\phi_{1}^{1}\right|_{\partial \Omega}=\left.\phi_{1}^{2}\right|_{\partial \Omega}=0 .
\end{aligned}
$$


Hence $\phi_{1}^{1}-\phi_{1}^{2}$ would be an harmonic function in $\Omega$ with $\phi_{1}^{1}-\phi_{1}^{2}=0$ in the boundary $\partial \Omega$, then by the minimum principle $\phi_{1}^{1}=\phi_{1}^{2}$, a contradiction. Assume that in the family of solutions $\left\{\phi_{k}\right\}_{k=1}^{\infty}$ the first $j$ functions $\phi_{1}, \phi_{2}, \ldots, \phi_{j}$ are unique but there exists two different $\phi_{j+1}^{1}$ and $\phi_{j+1}^{2}$ solutions in the $(j+1)$-th slot. Then $\triangle\left(\phi_{j+1}^{1}-\phi_{j+1}^{2}\right)=0$ and $\phi_{j+1}^{1}-\phi_{j+1}^{2}=0$ on $\partial \Omega$. Then $\phi_{j+1}^{1}=\phi_{j+1}^{2}$ Now, functions defined by $\phi_{k}(x)=k G\left(\phi_{k-1}\right)(x), k=1, \ldots$ are the solutions of the problem (1.1). Since the solution to the problem (1.1) is unique we only have to check that

$$
\triangle\left(k G\left(\phi_{k-1}\right)\right)=-k \phi_{k-1} .
$$

But that is straightforward because the Green operator is the inverse of $-\triangle$. To prove (3.10) let us use the induction method. Observe that $\phi_{1}=G(1)(x)$. Suppose that equation (3.10) is true and let us compute $\phi_{k+1}(x)$.

$$
\begin{aligned}
\phi_{k+1}(x) & =(k+1) G\left(\phi_{k}\right)(x)=(k+1) G\left(k ! G^{k}(1)\right)(x) \\
& =(k+1) k ! G\left(G^{k}(1)\right)(x)=(k+1) ! G^{k+1}(1)(x) .
\end{aligned}
$$

This proves (3.2) in Theorem 3.2. On the other hand, by (2.18),

$$
G^{k}(1)(x)=\sum_{i=1}^{\infty} \frac{a_{i} u_{i}(x)}{\lambda_{i}^{k}(\Omega)},
$$

where $a_{i}=\int_{\Omega} u_{i}(y) d \mu(y)$. Thus

$$
\phi_{k}(x)=k ! G^{k}(1)=k ! \sum_{i=1}^{\infty} \frac{a_{i} u_{i}(x)}{\lambda_{i}^{k}(\Omega)} .
$$

Moreover, the $L^{1}(\Omega, \mu)$-moment spectrum of $\Omega$ is readily obtained by

$$
\mathcal{A}_{k}(\Omega)=\int_{\Omega} \phi_{k} d \mu=k ! \sum_{i=1}^{\infty} \frac{a_{i}^{2}}{\lambda_{i}^{k}(\Omega)} .
$$

This proves Theorem 3.2 .

\section{4. $L^{2}$-expansion of the Green function $g(x, y)$}

Let $\left(\Omega, d s^{2}, \mu\right)$ be a relatively compact open subset of a Riemannian weighted manifold $\left(M, d s^{2}, \mu\right)$ and let $\sigma(\Omega, \mu)=\left\{\lambda_{k}\right\}_{k=1}^{\infty} \subset[0, \infty)$ be the spectrum of $\mathcal{L}=-\left.\triangle_{\mu}\right|_{W_{0}^{2}(\Omega, \mu)}$, repeated accordingly to their multiplicity. Let $\left\{u_{k}\right\}_{k=1}^{\infty}$ be an orthonormal basis in $L^{2}(\Omega, \mu)$ such that each function $u_{k}$ is an eigenfunction of $\mathcal{L}$ with eigenvalue $\lambda_{k}$. In this basis, the heat kernel $p_{t}^{\Omega}(x, y)$ of the operator $\mathcal{L}$ admits an expansion

$$
p_{t}^{\Omega}(x, y)=\sum_{k=1}^{\infty} e^{-\lambda_{k} t} u_{k}(x) u_{k}(y)
$$


This series converges absolutely and uniformly in the domain $t \geq \epsilon, x, y \in \Omega$ for any $\epsilon>0$ as well as in the topology of $C^{\infty}\left(\mathbb{R}_{+} \times \Omega \times \Omega\right)$, (see the details in [22, Thm. 10.13]). If we could integrate the heat kernel expansion (4.1) in the variable $t$ we would obtain that

$$
g^{\Omega}(x, y)=\sum_{k=1}^{\infty} \frac{u_{k}(x) \cdot u_{k}(y)}{\lambda_{k}(\Omega)} .
$$

The identity (4.2) holds in the sense of distributions, (see [22, exercise 13.14]) and we may ask whether this identity holds in stronger topology, for instance in $L^{1}(\Omega \times \Omega, \mu)$ or $L^{2}(\Omega \times \Omega, \mu)$ ? It is well known that $g^{\Omega}(x, y) \in L^{1}(\Omega \times \Omega, \mu)$, (see for instance, [22, Exercise 13.7]), however, $g^{\Omega}(x, \cdot)$ does not need to belong to $L^{2}(\Omega, \mu)$. Indeed, let $\Omega=B_{\mathbb{R}^{4}}(1) \subset \mathbb{R}^{4}$ to be the geodesic ball of the 4-dimensional Euclidean space $\mathbb{R}^{4}$, with its canonical metric, of radius $r=1$ and center at the origin 0 . The Green function of $\Omega$ is given by

$$
g^{\Omega}(0, y)=\frac{1}{2 \omega_{4}}\left(\frac{1}{|y|^{2}}-1\right),
$$

which obviously does not belong to $L^{2}\left(B_{\mathbb{R}^{4}}(1)\right)$. Here $\omega_{4}$ is the volume of the unit 3 -sphere in $\mathbb{R}^{4}$. On the other hand, if $\Omega=B_{\mathbb{R}}(1)$ is the geodesic ball of radius $r=1$ in the real line $\mathbb{R}$ then the Green function is $g^{\Omega}(x, y)=(|x-y|-x \cdot y+1) / 2$ which clearly is in $L^{2}(\Omega \times \Omega)$. We start showing that a necessary and sufficient condition to $g^{\Omega} \in L^{2}(\Omega \times \Omega, \mu)$, for any measure $\mu$, is that $\operatorname{dim}(M)=1,2,3$ and in these dimensions identity (4.2) holds in $L^{2}(\Omega \times \Omega, \mu)$. Precisely, we have the following result.

ThmMainA Theorem 4.1 Let $\left(M, d s^{2}, \mu\right)$ be a weighted Riemannian manifold and let $\Omega \subset M$ be a bounded open subset of $M$ with smooth boundary $\partial \Omega \neq \emptyset$. Then the Green function for the weighted Laplacian $g^{\Omega} \in L^{2}(\Omega \times \Omega, \mu)$ if and only if $\operatorname{dim}(M)=$ 1,2,3. Furthermore,

eqGreenSeries

$$
g^{\Omega}(x, y)=\sum_{k=1}^{\infty} \frac{u_{k}(x) \cdot u_{k}(y)}{\lambda_{k}(\Omega)},
$$

where the series converges in $L^{2}(\Omega \times \Omega, \mu)$, and $\left\{u_{k}\right\}_{k=1}^{\infty}$ is an orthonormal basis formed by eigenfunctions associated to the eigenvalues $\left\{\lambda_{k}(\Omega)\right\}_{k=1}^{\infty}$ of $\Omega$. Moreover

$$
\left\|g^{\Omega}\right\|_{L^{2}(\Omega \times \Omega)}=\sum_{k=1}^{\infty} \frac{1}{\lambda_{k}^{2}(\Omega)} .
$$

If $n=1$ then

$$
\int_{\Omega} g^{\Omega}(x, x) d \mu(x)=\sum_{k=1}^{\infty} \frac{1}{\lambda_{k}(\Omega)} .
$$

As mentioned before, this result above is a direct consequence of Weyl's asymptotic formula for the eigenvalues $\lambda_{i}(\Omega)$. In dimension one, it is a particular case of 
Mercer's Theorem (see [14, Chapter 3, Sec 4]). If $\Omega \subset \mathbb{R}^{n}, n=2,3$, this expansion is known to converge in $L^{2}$-sense, (see [14, Chapt. 5, Sec. 5]) and its proof can naturally adapted to domains in Riemannian manifolds, since it follows from Weyl's asymptotic formula. The first consequence of Theorem 4.1 is related to a result due to Grüter and Widman, see [24, Thm. 1.1]. They proved that for any $y \in \Omega \subset \mathbb{R}^{n}, n \geq 3$, the function

$$
x \rightarrow g^{\Omega}(x, y) \in L_{\frac{n}{n-2}}^{*}(\Omega),
$$

where $L_{p}^{*}(\Omega)$ is defined by

$$
L_{p}^{*}(\Omega):=\left\{f: \Omega \rightarrow \mathbb{R} \cup\{\infty\}, f \text { measurable and }\|f\|_{L_{p}^{*}(\Omega)}<\infty\right\}
$$

with

$$
\|f\|_{L_{p}^{*}(\Omega)}:=\sup _{t>0} t \cdot \nu(\{x \in \Omega:|f(x)|>t\})^{1 / p} .
$$

It is easy to see that $L^{p}(\Omega) \subset L_{p}^{*}(\Omega)$, thus coupling Theorem 4.1 with Grüter and Widman's result we obtain a more precise statement.

Corollary 4.2 Let $\Omega \subset \mathbb{R}^{n}, n \geq 3$ be a bounded open subset with smooth boundary. Let $g^{\Omega}$ to be the Green function (associated to the Laplacian $\triangle$ ).

- If $n=3$ then $x \rightarrow g^{\Omega}(x, y) \in L^{2}(\Omega) \cap L_{3}^{*}(\Omega), y \in \Omega$.

- If $n \geq 4$ then $x \rightarrow g^{\Omega}(x, y) \in L_{\frac{n}{n-2}}^{*}(\Omega) \backslash L^{2}(\Omega), y \in \Omega$.

Remark 4.3 In general, $L_{3}^{*}(\Omega) \backslash L^{2}(\Omega) \neq \emptyset$ and $L^{2}(\Omega) \backslash L_{3}^{*}(\Omega) \neq \emptyset$. Indeed, letting $\Omega=B_{r}(o) \subset \mathbb{R}^{3}$ and $f_{\alpha}(x)=1 /|x|^{\alpha}$ we have that $f_{\alpha} \in L_{3}^{*}(\Omega) \backslash L^{2}(\Omega)$ if $\alpha>1$ and $f_{\alpha} \in L^{2}(\Omega) \backslash L_{3}^{*}(\Omega)$ if $0<\alpha<1 / 2$.

4.0.1. Proof of Theorem 4.1. Let $\left(\Omega, d s^{2}, \mu\right)$ be a bounded weighted open subset of a weighted $m$-manifold $\left(M, d s^{2}, \mu\right)$ with smooth boundary $\partial \Omega \neq \emptyset$. Let $\sigma(\Omega)=\left\{\lambda_{i}(\Omega)\right\}_{i=1}^{\infty}$ be the sequence of eigenvalues of $\mathcal{L}=-\left.\triangle_{\mu}\right|_{W_{0}^{2}(\Omega, \mu)}$, repeated according to multiplicity. One has that the Weyl's asymptotic formula holds for the eigenvalues $\lambda_{i}$,

$$
\lambda_{i}(\Omega) \approx c_{m} \cdot\left(\frac{i}{\mu(\Omega)}\right)^{2 / m} \text { as } i \rightarrow \infty,
$$

where $c_{m}>0$ is the same constant as in $\mathbb{R}^{m}$, depending only on the dimension $m=\operatorname{dim}(M)$, see $[21, \mathrm{p} .7]$. Thus

$$
\sum_{i=1}^{\infty} \frac{1}{\lambda_{i}^{2}(\Omega)}<\infty \Leftrightarrow m=1,2,3
$$

and

$$
\sum_{i=1}^{\infty} \frac{1}{\lambda_{i}(\Omega)}<\infty \Leftrightarrow m=1
$$


Let $\left\{g_{k}: \Omega \times \Omega \rightarrow \mathbb{R}\right\} \subset L^{2}(\Omega \times \Omega, \mu)$ be a sequence of functions defined by

$$
g_{k}(x, y)=\sum_{i=1}^{k} \frac{u_{i}(x) u_{i}(y)}{\lambda_{i}(\Omega)}
$$

where $\left\{u_{i}\right\}$ is the complete and orthonormal basis of $L^{2}(\Omega, \mu)$, formed by eigenfunctions $u_{i}$ corresponding to the eigenvalues $\lambda_{i}(\Omega)$. Let $k_{2}>k_{1}$ and compute

$$
\left\|g_{k_{2}}-g_{k_{1}}\right\|_{L^{2}(\Omega \times \Omega, \mu)}^{2}=\sum_{i=k_{1}+1}^{k_{2}} \frac{1}{\lambda_{i}^{2}(\Omega)} .
$$

The sequence $\left\{g_{k}\right\}$ is a Cauchy sequence in $L^{2}(\Omega \times \Omega, \mu)$ iff $\sum_{i=1}^{\infty} \frac{1}{\lambda_{i}^{2}(\Omega)}<\infty$. Then, $g_{k} \rightarrow g_{\infty}$ in $L^{2}(\Omega \times \Omega, \mu)$ if and only if $\operatorname{dim}(M)=1,2,3$, where

$$
g_{\infty}(x, y)=\sum_{i=1}^{\infty} \frac{u_{i}(x) u_{i}(y)}{\lambda_{i}(\Omega)} \in L^{2}(\Omega \times \Omega, \mu) .
$$

On the other hand, the Green function $g^{\Omega}$ satisfies the functional identity

$$
g^{\Omega}(x, y)=\sum_{i=1}^{\infty} \frac{u_{i}(x) u_{i}(y)}{\lambda_{i}(\Omega)}
$$

in the sense of distributions, see [22, p. 348]. Therefore, $g^{\Omega}=g_{\infty} \in L^{2}(\Omega \times \Omega, \mu)$ if and only if $m=1,2,3$ and

$$
\left\|g^{\Omega}\right\|_{L^{2}(\Omega \times \Omega, \mu)}^{2}=\sum_{i=1}^{\infty} \frac{1}{\lambda_{i}^{2}(\Omega)}<\infty .
$$

In the case when $m=1$ the sequence $g_{k}(x)=\sum_{i=1}^{k} \frac{u_{i}^{2}(x)}{\lambda_{i}(\Omega)}$ converges pointwise to $g^{\Omega}(x, x)=\sum_{i=1}^{\infty} \frac{u_{i}^{2}(x)}{\lambda_{i}(\Omega)}$, since $g^{\Omega} \in L^{1}(\Omega, \mu)$ and $\left|g_{k}\right| \leq g$ by the Lebesgue dominated convergence theorem

$$
\sum_{i=1}^{\infty} \frac{1}{\lambda_{i}(\Omega)}=\lim _{k \rightarrow \infty} \int_{\Omega} g_{k}(x) d \mu=\int_{\Omega} g^{\Omega}(x, x) d \mu .
$$

\section{Spectrum of rotationally invariant balls}

A rotationally invariant Riemannian $m$-manifold, also called a model manifold, with radial sectional curvature $-G(r)$ along the geodesic issuing from the origin, where $G: \mathbb{R} \rightarrow \mathbb{R}$ is a smooth even function, is the quotient space

$$
\mathbb{M}_{h}^{m}=\left[0, R_{h}\right) \times \mathbb{S}^{m-1} / \sim
$$


with $(t, \theta) \sim(s, \beta) \Leftrightarrow t=s=0$ or $t=s$ and $\theta=\beta$, endowed with the metric $d s_{h}^{2}(t, \theta)=d t^{2}+h^{2}(t) d \theta^{2}$ where $h:[0, \infty) \rightarrow \mathbb{R}$ is the unique solution of the Cauchy problem

$$
\left\{\begin{array}{l}
h^{\prime \prime}-G h=0 \\
h^{\prime}(0)=1, h^{(2 k)}(0)=0, k=0,1, \ldots,
\end{array}\right.
$$

and $R_{h}$ is the largest positive real number such that $\left.h\right|_{\left(0, R_{h}\right)}>0$. If $R_{h}=\infty$, the manifold $\mathbb{M}_{h}^{m}$ is geodesically complete and non-compact with a pole in the origin. The geodesic ball $B_{h}(o, r)$ centered at the origin $o=\{0\} \times \mathbb{S}^{m-1} / \sim$ with radius $r<R_{h}$, i.e. the set $[0, r) \times \mathbb{S}^{m-1} / \sim$, is rotationally invariant. The volume $V(r)$ of the ball $B_{h}(o, r)$ and the volume $S(r)$ of the boundary $\partial B_{h}(o, r)$ are given by

$$
V(r)=\omega_{m} \int_{0}^{r} h^{m-1}(s) d s \quad \text { and } \quad S(r)=\omega_{m} h^{m-1}(r),
$$

respectively, where $\omega_{m}=\operatorname{vol}\left(\mathbb{S}^{m-1}\right)$. The Laplace operator on $B_{h}(o, r)$, expressed in polar coordinates, is given by

$$
\triangle=\frac{\partial^{2}}{\partial t^{2}}+(m-1) \frac{h^{\prime}}{h} \frac{\partial}{\partial t}+\frac{1}{h^{2}} \triangle_{\theta} .
$$

To search for the Dirichlet eigenvalues $\lambda$ of $B_{h}(o, r)$ it is enough to seek smooth functions of the form $u(t, \theta)=T(t) H(\theta)$ satisfying $\triangle u+\lambda u=0$ in $B_{h}(o, r)$ and $u \mid \partial B_{h}(o, r)=0$, see [11, p. 42]. This is equivalent to the following eigenvalue problems

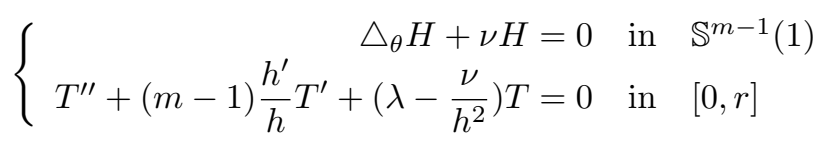

with initial conditions $T^{\prime}(0)=0$ if $l=0, T(t) \sim c \cdot t^{l}$ as $t \rightarrow 0$ when $l=1,2 \ldots$ and $T(r)=0$. Here $T^{\prime}=\partial T / \partial t, T^{\prime \prime}=\partial^{2} T / \partial t^{2}$.

The eigenvalues of the sphere are given by $\nu_{l}=l(l+m-2), l=0,1, \ldots$ For each value $\nu_{l}$, the set of all $\lambda$ such that the equation

$$
T^{\prime \prime}+(m-1) \frac{h^{\prime}}{h} T^{\prime}+\left(\lambda-\frac{\nu_{l}}{h^{2}}\right) T=0
$$

has a non-trivial solution satisfying the initial conditions consist of an increasing sequence of positive real numbers $\left\{\lambda_{l, j}\right\}_{j=1}^{\infty}$,

$$
0 \leq \lambda_{l, 1}<\lambda_{l, 2}<\cdots \uparrow+\infty .
$$

Moreover, each $\lambda_{l, i}$ determines a 1-dimensional space of solutions, say, generated by $T_{l, i}$. The sequence (5.4) is called the $\nu_{l}$-spectrum, without repetitions, of $B_{h}(o, r)$, denoted by $\sigma^{l}\left(B_{h}(o, r)\right)$. The multiplicity of each $\nu_{l}$ is given by

$$
\delta(l, m)=\left(\begin{array}{c}
m-1+l \\
l
\end{array}\right)-\left(\begin{array}{c}
m-2+l \\
l-1
\end{array}\right) .
$$


Thus, there exists an orthonormal basis formed by eigenfunctions $H_{l, 1}, \ldots, H_{l, \delta}$ of the vector space $V_{\nu_{l}}=\left\{\phi: \triangle_{\theta} \phi+\nu_{l} \phi=0\right\}$. This implies that the set of functions

$$
\left\{T_{l, i}(t) H_{l, 1}(\theta), T_{l, i}(t) H_{l, 2}(\theta), \ldots, T_{l, i}(t) H_{l, \delta}(\theta)\right\}
$$

is an orthonormal basis of the vector space $V_{\lambda_{l, i}}=\left\{\psi: \Delta \psi+\lambda_{l, i} \psi=0\right\}$. Thus, the multiplicity of each eigenvalue $\lambda_{l, i}$ in $(5.4)$, in the spectrum $\sigma\left(B_{h}(o, r)\right)$ is $\delta(l, m)$. Since all of the eigenvalues of $B_{h}(o, r)$ are obtained by the procedure above, the spectrum of $B_{h}(o, r)$, without repetitions, is the union of the $\nu_{l}$-spectrum $\sigma^{l}\left(B_{h}(o, r)\right), l=0,1, \ldots$

$$
\sigma\left(B_{h}(o, r)\right)=\cup_{l=0}^{\infty} \sigma^{l}\left(B_{h}(o, r)\right)=\left\{\lambda_{l, j}\right\}_{l=0, j=1}^{\infty, \infty}
$$

each $\lambda_{l, i}$ with multiplicity $\delta(l, m)$. i.e. $\operatorname{dim} V_{\lambda_{l, i}}=\delta(l, m)$. The details of this discussion can be found in [11, pp. 40-42].

The eigenfunctions associated to the eigenvalues $\lambda \in \sigma^{0}\left(B_{h}(o, r)\right)=\left\{\lambda_{0, i}\right\}$, $i=1,2 \ldots$ are (radial) eigenfunctions $u_{i}(t, \theta)=T_{0, i}(t)$. On the other hand, let us suppose that $\lambda \in \cup_{l=0}^{\infty} \sigma^{l}\left(B_{h}(o, r)\right)$ is an eigenvalue whose eigenfunction is radial, $u(t, \theta)=T_{l, i}(t) H_{l, i}(\theta)=c \cdot T_{l, i}(t)$, then the eigenfunction of the sphere $H_{l, i}(\theta)=c$ is constant then its eigenvalue is zero and $\lambda \in \sigma^{0}\left(B_{h}(o, r)\right)$. This shows that the set of "radial" eigenvalues is the $\sigma^{0}$-spectrum. This motivates the following definition.

Definition 5.1 Let $B(p, r) \subset M$ be a geodesic ball with center at $p$ and radius $r$. The radial spectrum $\sigma^{\mathrm{rad}}(B(p, r))$ of $B(p, r)$ is formed by those eigenvalues $\lambda_{k}$ of $\mathcal{L}=-\left.\triangle\right|_{W_{0}^{2}(B(p, r))}$ whose associated eigenspace $V_{k}$ contains only radial eigenfunctions.

The radial spectrum of a general geodesic ball may be empty. However, if $B(p, r)$ is rotationally invariant, then its radial spectrum is $\sigma^{\operatorname{rad}}(B(p, r))=\sigma^{0}(B(p, r))$. It should be remarked that there are non-rotationally invariant geodesic balls with non-empty radial spectrum, (see Example 5.3).

\subsection{Stochastically incomplete model manifolds}

Let $M$ be a Riemannian manifold and $p_{t}(x, y) \in C^{\infty}((0, \infty) \times M \times M)$ be the heat kernel of $M$. It is well known that

$$
\int_{M} p_{t}(x, y) d \nu(y) \leq 1
$$

This property allows one to see the heat kernel as a probability distribution in the space of Brownian paths on $M$. More precisely, for $x \in M$ and an open subset $U \subset M$, the quantity $\int_{U} p_{t}(x, y) d \nu(y)$ is the probability that a Brownian path $s \rightarrow X_{s}$ emanating from $X_{0}=x$ lies in $X_{t} \in U$ at time $t$. The strict inequality, $\int_{M} p_{t}(x, y) d \nu(y)<1$, implies that there is a positive probability that stochastic process $t \rightarrow X_{t}$ ends in finite time, i.e. the Brownian path reach infinity in finite time $t$. This motivates the following definition. 
Definition 5.2 A Riemannian manifold $M$ is stochastically complete if for every $x \in M$ and $t>0$ one has that

$$
\int_{M} p_{t}(x, y) d \nu(y)=1
$$

otherwise $M$ is said to be stochastically incomplete.

There exists a simple and elegant geometric criteria for stochastic incompleteness of model manifolds $\mathbb{M}_{h}^{m}$, proved by many authors in different settings.

Theorem 5.3 ([1], [19], [20], [26]) A geodesically complete model manifold $\mathbb{M}_{h}^{m}$ is stochastically incomplete if and only if

$$
\int_{0}^{\infty} \frac{V(t)}{S(t)} d t<\infty
$$

thm1.4

Where $V(r)=\omega_{m} \int_{0}^{r} h^{m-1}(s) d s$ and $S(r)=\omega_{m} h^{m-1}(r)$.

The spectrum of a stochastically incomplete model $m$-manifold $\mathbb{M}_{h}^{m}$ is discrete, (see [9, Example 6.12]). Let

$$
\sigma\left(\mathbb{M}_{h}^{m}\right)=\left\{0<\lambda_{1}\left(\mathbb{M}_{h}^{m}\right)<\lambda_{2}\left(\mathbb{M}_{h}^{m}\right) \leq \cdots\right\},
$$

be the spectrum of $\mathbb{M}_{h}^{m}$ with repetition and

$$
\sigma\left(B_{h}(o, r)\right)=\left\{0<\lambda_{1}\left(B_{h}(o, r)\right)<\lambda_{2}\left(B_{h}(o, r)\right) \leq \cdots\right\}
$$

be the spectrum of $B_{h}(o, r) \subset \mathbb{M}_{h}^{m}$ with repetition. It is well known that

$$
\lambda_{1}\left(\mathbb{M}_{h}^{m}\right)=\lim _{r \rightarrow \infty} \lambda_{1}\left(B_{h}(o, r)\right) .
$$

What is less known is that the $\mathrm{k}^{t h}$-element $\lambda_{k}\left(\mathbb{M}_{h}^{m}\right)$ of the sequence (5.6) is also obtained as a limit

$$
\lambda_{k}\left(\mathbb{M}_{h}^{m}\right)=\lim _{r \rightarrow \infty} \lambda_{k}\left(B_{h}(o, r)\right)
$$

for $k=1,2, \ldots$.

The identity (5.7) remains true for eigenvalues of more general operators as the weighted Laplacian $\triangle_{\mu}$, (see [10, Sec. 2.3 \& eq. 2.85]). In particular, it is true for the eigenvalues of the operator $L_{0}(T)=T^{\prime \prime}+(m-1) \frac{h^{\prime}}{h} T^{\prime}$ on $[0, r]$, since $L_{0}=\triangle_{\mu}$, for $d \mu=\omega_{m-1} h^{m-1} d r$, where $\omega_{m-1}=\operatorname{vol}\left(\mathbb{S}^{m-1}\right)$.

The radial spectrum of $B_{h}(o, r) \subset \mathbb{M}_{h}^{m}$,

$$
\sigma^{\mathrm{rad}}\left(B_{h}(o, r)\right)=\left\{0<\lambda_{1}^{\mathrm{rad}}\left(B_{h}(o, r)\right) \leq \lambda_{2}^{\mathrm{rad}}\left(B_{h}(o, r)\right) \leq \cdots\right\},
$$

is exactly the spectrum of the operator $L_{0}(T)=T^{\prime \prime}+(m-1) \frac{h^{\prime}}{h} T^{\prime}$ on $[0, r]$ and if $\mathbb{M}_{h}^{m}$ has discrete spectrum, the radial spectrum of $\mathbb{M}_{h}^{m}$ is exactly the spectrum of the operator $L_{0}$ on $[0, \infty)$. Therefore, the $\mathrm{i}^{\text {th }}$-radial eigenvalue of $\mathbb{M}_{h}^{m}$ is obtained as a limit

$$
\lambda_{i}^{\mathrm{rad}}\left(\mathbb{M}_{h}^{m}\right)=\lim _{r \rightarrow \infty} \lambda_{i}^{\mathrm{rad}}\left(B_{h}(o, r)\right)
$$

for $i=1,2, \ldots$. 


\section{2. "Harmonic series" of radial eigenvalues}

Our main result in Section 5 regards the radial spectrum of rotationally invariant balls of model manifolds. The radial spectrum of a stochastically incomplete model manifolds $\mathbb{M}_{h}^{m}$ is deeply related with this geometric criteria as shown by our first result, Theorem 5.4.

thmMain1-intro

Theorem 5.4 Let $B_{h}(o, r)$ be a geodesic ball of radius $r$ with $\lambda_{1}\left(B_{h}(o, r)\right)>0$ of a model $\mathbb{M}_{h}^{m}$. Let $\sigma^{\mathrm{rad}}\left(B_{h}(o, r)\right)=\left\{\lambda_{1}^{\mathrm{rad}}\left(B_{h}(o, r)\right)<\lambda_{2}^{\mathrm{rad}}\left(B_{h}(o, r)\right)<\cdots\right\}$ be the radial spectrum of $B_{h}(o, r)$. Then

eqMain1-intro

$$
\sum_{i=1}^{\infty} \frac{1}{\lambda_{i}^{\mathrm{rad}}\left(B_{h}(o, r)\right)}=\int_{0}^{r} \frac{V(t)}{S(t)} d t
$$

If the model $\mathbb{M}_{h}^{m}$ is stochastically incomplete then

eqMain2-intro

$$
\sum_{i=1}^{\infty} \frac{1}{\lambda_{i}^{\operatorname{rad}}\left(\mathbb{M}_{h}^{n}\right)}=\int_{0}^{\infty} \frac{V(t)}{S(t)} d t
$$

Where $V(t)=\omega_{m} \int_{0}^{t} h^{m-1}(s) d s$ and $S(t)=\omega_{m} h^{m-1}(t)$.

Corollary 5.5 Let $B(o, 1)$ be a geodesic ball of radius $r=1$ in the model manifold $\mathbb{M}_{h}^{2}$, where $h(t)=\sinh (t), t, \sin (t)$, respectively. Then,

$$
\begin{aligned}
& \sum_{i=1}^{\infty} \frac{1}{\lambda_{i}^{\mathrm{rad}}(B(o, 1))}=\log \left(\frac{(1+e)^{2}}{4 e}\right) \approx 0.240229 \text { if } h(t)=\sinh (t) \\
& \sum_{i=1}^{\infty} \frac{1}{\lambda_{i}^{\mathrm{rad}}(B(o, 1))}=0.25 \text { if } h(t)=t \\
& \sum_{i=1}^{\infty} \frac{1}{\lambda_{i}^{\mathrm{rad}}(B(o, 1))}=\log \left(\sec \left(\frac{1}{2}\right)\right) \approx 0.261168 \text { if } h(t)=\sin (t)
\end{aligned}
$$

When the model manifold $\mathbb{M}_{h}^{m}$ is the Euclidean space $\mathbb{R}^{m}$, the "harmonic series" of the eigenvalues $\left\{\lambda_{l, i}\right\}_{i=1}^{\infty}=\sigma^{l}\left(B_{h}(o, r)\right)$, (without repetitions), for $l=0,1, \ldots$, also converges.

thmMain2 Theorem 5.6 Let $B_{h}(o, r)$ be the geodesic ball of $\mathbb{R}^{m}$ with radius $r$ centred at the origin o. Let $\sigma^{l}\left(B_{h}(o, r)\right)=\left\{\lambda_{l, 1}\left(B_{h}(o, r)\right)<\lambda_{l, 2}\left(B_{h}(o, r)\right)<\cdots\right\}$ be the $\nu_{l}$-spectrum of $B_{h}(o, r)$ without repetition, $l=0,1, \ldots$ Then

eqMain3

$$
\sum_{i=1}^{\infty} \frac{1}{\lambda_{l, i}\left(B_{h}(o, r)\right)}=\left(\frac{1}{1+2 \frac{l}{m}}\right) \int_{0}^{r} \frac{V(t)}{S(t)} d t=\left(\frac{1}{1+2 \frac{l}{m}}\right) \frac{r^{2}}{2 m}
$$

and

$$
\sum_{i=1}^{\infty} \frac{1}{\lambda_{l, i}^{2}\left(B_{h}(o, r)\right)}=\frac{r^{4}}{2(2 l+m)^{2}(2+2 l+m)}
$$


If we let $\sigma\left(B_{h}(o, r)\right)=\left\{0<\lambda_{1}<\lambda_{2} \leq \cdots\right\}$ be the spectrum of be $B_{h}(o, r) \subset$ $\mathbb{R}^{m}$, repeating the eigenvalues according to their multiplicities, in this case, $\sigma\left(B_{h}(o, r)\right)$ is a union of $\delta(l, m)$-copies of $\sigma^{l}\left(B_{h}(o, r)\right)$ for $l=0,1, \ldots$, then we can compute the whole sum

$$
\sum_{k=1}^{\infty} \frac{1}{\lambda_{k}^{2}}=\sum_{l=0}^{\infty}\left(\sum_{i=1}^{\infty} \frac{\delta(l, m)}{\lambda_{l, i}^{2}}\right)=\sum_{l=0}^{\infty} \delta(l, m) \cdot \frac{r^{4}}{2(2 l+m)^{2}(2+2 l+m)} .
$$

In the particular case of dimension $m=2$ or dimension $m=3$,

$$
\begin{aligned}
\sum_{l=0}^{\infty} \delta(l, 2) \cdot \frac{r^{4}}{2(2 l+2)^{2}(2+2 l+2)} & =\frac{\pi^{2}-6}{96} \cdot r^{4} \\
\sum_{l=0}^{\infty} \delta(l, 3) \cdot \frac{r^{4}}{2(2 l+3)^{2}(5+2 l)} & =\frac{12-\pi^{2}}{64} \cdot r^{4}
\end{aligned}
$$

However, this series diverges for $m \geq 4$ in agreement with Theorem 4.1 equation (4.4). The convergence of the series is related to the fact that the Green function belongs to $L^{2}$ only in the case of $m=1,2,3$. We should remark that the divergence in higer dimension is because of the multiplicity of the eigenvalues. In fact, if we consider the spectrum $\widetilde{\sigma}\left(B_{h}(o, r)\right)=\cup_{l=1}^{\infty} \sigma^{l}\left(B_{h}(o, r)\right)=\left\{0<\bar{\lambda}_{1}<\bar{\lambda}_{2}<\cdots\right\}$ without repetition then

$$
\sum_{k=1}^{\infty} \frac{1}{\tilde{\lambda}_{k}^{2}}=\sum_{l=0}^{\infty}\left(\sum_{i=1}^{\infty} \frac{1}{\lambda_{l, i}^{2}}\right)=\sum_{l=0}^{\infty} \frac{r^{4}}{2(2 l+m)^{2}(2+2 l+m)}<\infty .
$$

Morever, $\lim _{m \rightarrow \infty} \sum_{k=1}^{\infty} \frac{1}{\widetilde{\lambda}_{k}^{2}}=0$.

cor2.2 Corollary 5.7 Let $B_{h}(o, r)$ be the geodesic ball of $\mathbb{R}^{m}$ with radius $r$ centred at the origin o . Let $\sigma^{l}\left(B_{h}(o, r)\right)=\left\{\lambda_{l, 1}\left(B_{h}(o, r)\right) \leq \lambda_{l, 2}\left(B_{h}(o, r)\right) \leq \ldots\right\}, l=0,1, \ldots$ Then

$$
\lambda_{l, k}\left(B_{h}(o, r)\right) \geq \frac{k(m+2 l)}{m} \cdot \frac{1}{\int_{0}^{r} \frac{V(s)}{S(s)} d s}=\frac{2 k(m+2 l)}{r^{2}} .
$$

Corollary 5.7 should be compared with the estimates obtained in [5, Thm.2.1] and [8, Thm.2.7] for the first eigenvalue. We believe that Theorem 4.1 is also valid for rotationally invariant geodesic balls of model manifolds. We formalize this in the following conjecture

Conjecture 5.8 There exists a constant $c=c(m, l)<1$ such that for a geodesic ball $B_{h}(o, r) \subset \mathbb{M}_{h}^{m}$ the harmonic series converges

$$
\sum_{i=1}^{\infty} \frac{1}{\lambda_{l, i}\left(B_{h}(o, r)\right)}=c \cdot \int_{0}^{r} \frac{V(s)}{S(s)} d s, l=0,1,2, \ldots
$$




\subsection{Example}

Let $\{\partial / \partial x, \partial / \partial y, \partial / \partial z\}$ be a globally defined non-zero vector fields on $\mathbb{S}^{3}$ satisfying these conditions

$$
[\partial / \partial x, \partial / \partial y]=2 \partial / \partial z,[\partial / \partial y, \partial / \partial z]=2 \partial / \partial x,[\partial / \partial z, \partial / \partial x]=2 \partial / \partial y
$$

and let $d x, d y$ and $d z$ be their dual 1-forms. Consider, on $\Omega=[0, r] \times \mathbb{S}^{3} / \sim$, where $(t, \theta) \sim(s, \beta) \Leftrightarrow t=s=0$ or $t=s$ and $\theta=\beta$, the following metric

$$
d s^{2}=d t^{2}+a^{2}(t, \theta) d x^{2}+b^{2}(t, \theta) d y^{2}+c^{2}(t, \theta) d z^{2},
$$

where $a, b, c:[0, r] \times \mathbb{S}^{3} \rightarrow \mathbb{R}$ are defined by

$$
\begin{aligned}
& a(t, \theta)=\sinh ^{2}[t] / t \cdot f(t, \theta) \\
& b(t, \theta)=t \cdot f(t, \theta) \\
& c(t, \theta)=\sinh [t]
\end{aligned}
$$

with $f:[0, r] \times \mathbb{S}^{3} \rightarrow(0, \infty)$ given by $f(t, \theta)=1+t^{k} q(\theta), k \geq 3$ and $q: \mathbb{S}^{3} \rightarrow(0, \infty)$ is smooth. This metric is clearly smooth in $(0, r] \times \mathbb{S}^{3} / \sim$. We need only check that it is smooth at the origin $\{0\} \times \mathbb{S}^{3} \sim$. The coefficients near $t=0$ and every $\theta \in \mathbb{S}^{3}$ fixed are given by $a(t, \theta)=t+t^{3} / 3+2 t^{5} / 45+O\left(t^{6}\right), b(t, \theta)=t+t^{3} / 6+t^{5} / 120+O\left(t^{6}\right)$, $c(t, \theta)=t+O\left(t^{6}\right)$. This shows that $d s^{2} \approx \mathrm{can}_{\mathbb{H}^{4}}$ in the $C^{2}$-topology as $t \approx 0$, where $\operatorname{can}_{\mathbb{H}^{4}}=d t^{2}+\sinh ^{2}[t]\left(d x^{2}+d y^{2}+d z^{2}\right)$ is the canonical metric on the hyperbolic space $\mathbb{H}^{4}(-1)$. Let $\Omega=B_{h}(o, r)$ be the geodesic ball with center at the origin $o=\{0\} \times \mathbb{S}^{3} / \sim$ and radius $r$ with respect to the metric $d s^{2}$. It is clear that $\Omega$ is not rotationally symmetric. The Laplace operator $\triangle_{d s^{2}}$ is given by

$$
\begin{aligned}
\triangle_{d s^{2}}(t, \theta)= & \frac{\partial^{2}}{\partial t^{2}}+3 \operatorname{coth}(t) \frac{\partial}{\partial t} \\
& +\left[\frac{t^{2}\left(1+t^{k} q(\theta)\right)^{2}}{\sinh ^{4}(t)}\right] \frac{\partial^{2}}{\partial x^{2}}+\left[\frac{1}{t^{2}\left(1+t^{k} q(\theta)\right)^{2}}\right] \frac{\partial^{2}}{\partial y^{2}}+\frac{1}{\sinh ^{2}(t)} \frac{\partial^{2}}{\partial z^{2}} \\
& +\left[\frac{2 t^{2+k}\left(1+t^{k} q(\theta)\right)}{\sinh ^{2}(t)}\right] \frac{\partial q}{\partial x} \frac{\partial}{\partial x}-\left[\frac{2 t^{k-2}}{\left(1+t^{k} q(\theta)\right)^{3}}\right] \frac{\partial q}{\partial y} \frac{\partial}{\partial y} .
\end{aligned}
$$

Let $\widetilde{\Omega}=\left([0, r) \times \mathbb{S}^{3} / \sim, \operatorname{can}_{\mathbb{H}^{4}}\right)$ be the geodesic ball of radius $r$ centered at the origin in the Hyperbolic space $\mathbb{H}^{4}(-1)$. The Laplace operator $\triangle_{\text {can }_{\mathbb{H}}}$ on $\widetilde{\Omega}$ is given by

$$
\triangle_{\operatorname{can}_{\mathbb{H}} 4}(t, \theta)=\frac{\partial^{2}}{\partial t^{2}}+3 \operatorname{coth}(t) \frac{\partial}{\partial t}+\frac{1}{\sinh ^{2}(t)}\left(\frac{\partial^{2}}{\partial x^{2}}+\frac{\partial^{2}}{\partial y^{2}}+\frac{\partial^{2}}{\partial z^{2}}\right) .
$$

Observe that $\triangle_{d s^{2}}=\triangle_{\operatorname{can}_{\mathbb{H}}}$ on the set of the smooth radial functions $u(t, \theta)=u(t)$ with $u^{\prime}(0)=0$, in particular, $\Omega$ and $\widetilde{\Omega}$ has the same radial eigenfunctions and radial eigenvalues. 
Hence, $\left(\Omega, d s^{2}\right)$ is a non-rotationally symmetric geodesic ball with the same radial spectrum of a rotationally symmetric geodesic ball $\left(\tilde{\Omega}, \operatorname{can}_{\mathbb{H}^{4}}\right)$. Note that the functions $t \rightarrow V(t)$ and $t \rightarrow S(t)$ are the same for both metrics, which can be checked directly, coherently with the identity (5.8). The example (5.3) is a variation of the example [7, Examp. 2] which, by its turn, was havily based in the example of G. Perelman in [35].

5.3.1. Proof of Theorem 5.4. The metric on the geodesic ball $B_{h}(o, r) \subset \mathbb{M}_{h}^{n}$ is expressed, in polar coordinates, as $d s^{2}=d t^{2}+h^{2}(t) d \theta^{2}$. The Laplacian $\triangle$ of this metric is given by

$$
\begin{aligned}
\triangle(t, \theta) & =\frac{\partial^{2}}{\partial t^{2}}+(n-1) \frac{h^{\prime}}{h}(t) \frac{\partial}{\partial t}+\frac{1}{h^{2}}(t) \triangle_{\theta} \\
& =L_{0}+\frac{1}{h^{2}}(t) \triangle_{\theta},
\end{aligned}
$$

and $\triangle_{\theta}$ is the Laplacian on $\mathbb{S}^{n-1}$. Observe that the radial eigenvalues of $B_{h}(o, r)$ are the eigenvalues of the operator $L_{0}$ in the following eigenvalue problem.

$$
\left\{\begin{aligned}
L_{0} u+\lambda u & =0 \\
u^{\prime}(0) & =0 \\
u(r) & =0 .
\end{aligned}\right.
$$

In order to study this eigenvalue problem, define the following space of functions

$$
\Lambda:=\left\{u \in W^{2}([0, r], \mu): \lim _{t \rightarrow 0^{+}} u^{\prime}(t)=0 \text { and } u(r)=0\right\}
$$

and the density in $[0, r]$ given by $d \mu(t)=\omega_{n} h^{n-1}(t) d t$. Observe that $f \in L^{2}([0, r], \mu)$ if and only if $f \circ t \in L^{2}\left(B_{h}(o, r)\right)$. Define the bilinear form $\mathcal{E}_{b f}$ acting on Lipschitz functions

$$
\mathcal{E}_{b f}(f, g)=\int_{0}^{r} f^{\prime}(s) g^{\prime}(s) d s
$$

and let $\mathcal{F}$ be the closure of $\Lambda$ in $L^{2}([0, r], \mu)$ with respect to the norm

$$
\|f\|_{\mathcal{F}}^{2}=\|f\|_{L^{2}([0, r], \mu)}^{2}+\mathcal{E}_{b f}(f, f)
$$

The bilinear form $\mathcal{E}_{b f}$ acting on $\mathcal{F}$, in the distributional sense, is a Dirichlet form, i.e. it has the following properties.

1. Positivity: $\mathcal{E}_{b f}(f)=\mathcal{E}_{b f}(f, f) \geq 0$ for any $f \in \mathcal{F}$.

2. Closedness: the space $\mathcal{F}$ is a Hilbert space with respect to the following product

$$
\langle f, g\rangle=(f, g)+\mathcal{E}_{b f}(f, g) .
$$

3. The Markov property: if $f \in \mathcal{F}$ then the function

$$
g:=\min \{1, \max \{f, 0\}\}
$$

also belongs to $\mathcal{F}$ and $\mathcal{E}_{b f}(g) \leq \mathcal{E}_{b f}(f)$. Here we used the shorthand notation $\mathcal{E}_{b f}(f):=\mathcal{E}_{b f}(f, f)$. 
Any Dirichlet form $\mathcal{E}_{b f}$ has a generator $\mathcal{L}$ which is a non-positive definite selfadjoint operator on $L^{2}([0, r], \mu)$ with domains $\mathcal{D}=\mathcal{D}(\mathcal{L}) \subset \mathcal{F}$ such that

$$
\mathcal{E}_{b f}(f, g)=(-\mathcal{L}(f), g)
$$

for $f \in \mathcal{D}$ and $g \in \mathcal{F}$ where $\mathcal{D}$ is dense in $\mathcal{F}$, see details in [23, Sec.2.2].

prop7.1 Proposition 5.9 The operator $\left.\mathcal{L}\right|_{\mathcal{D}}$ is an extension of $\left.L_{0}\right|_{\Lambda}$, that is,

$$
\mathcal{L} f=L_{0} f \text {, for any } f \in \Lambda .
$$

Proof: For any $f, g \in \Lambda$

$$
\begin{aligned}
(\mathcal{L} f, g) & =-\mathcal{E}_{b f}(f, g) \\
& =-\omega_{n} \int_{0}^{r} f^{\prime}(t) g^{\prime}(t) h^{n-1}(t) d t \\
& =-\omega_{n} \int_{0}^{r}\left[\frac{d}{d t}\left(f^{\prime}(t) g(t) h^{n-1}(t)\right)-g(t) \frac{d}{d t}\left(f^{\prime}(t) h^{n-1}(t)\right)\right] d t \\
& =-\omega_{n}\left[f^{\prime}(t) g(t) h^{n-1}(t)\right]_{0}^{r}+\int_{0}^{r} g(t) L_{0} f(t) d \mu(t) \\
& =\left(L_{0} f, g\right) .
\end{aligned}
$$

The generator $\mathcal{L}$ determines the heat semigroup $P_{t}=e^{-\mathcal{L} t}, t \geq 0$ which posses a heat kernel $p(t, x, y)$ and Green function $g(x, y)=\int_{0}^{\infty} p(t, x, y) d t$. Observe that $\left.\mathcal{L}\right|_{\mathcal{D}}$ is a self-adjoint extension of $\left.L_{0}\right|_{\Lambda}$. Thus, the solution of eigenvalue problem (5.15) is an infinite sequence of eigenvalues $0<\lambda_{1}^{\text {rad }}<\lambda_{2}^{\text {rad }}<\cdots \rightarrow \infty$, (the radial spectrum of $\left.B_{h}(o, r)\right)$. Observing that $L_{0}=\triangle_{\mu}$, for $d \mu(t)=\omega_{n} h^{n-1}(t)$, we have by Theorem 4.1 that

eq6.15

$$
\sum_{i=1}^{\infty} \frac{1}{\lambda_{i}^{\mathrm{rad}}\left(B_{h}(o, r)\right)}=\int_{0}^{r} g(x, x) d \mu(x) .
$$

We need to determine the Green function $g(x, y)$ for the operator $\mathcal{L}$.

prop-green-rad

green-rad1
Proposition 5.10 The Green function $g(x, y)$ for the operator $\mathcal{L}$ is given by

$$
g(x, y)=\int_{x}^{r} \frac{1}{\omega_{n} h^{n-1}(t)} \theta_{y}(t) d t,
$$

where

$$
\theta_{y}(t):=\left\{\begin{array}{lll}
1 & \text { if } \quad t \geq y \\
0 & \text { if } \quad t<y
\end{array}\right.
$$

Moreover,

$$
G(f)(x)=\int_{0}^{r} g(x, y) f(y) d \mu(y)=T(f)(x) .
$$

Here $T$ is the operator defined in (2.10). 
Proof: We need to prove that $g(r, y)=0, \lim _{x \rightarrow 0^{+}} \frac{\partial}{\partial x} g(x, y)=0, \mathcal{L}_{x} g(x, y)=0$ for $x \neq y$ and more generally $\mathcal{L} g(x, \cdot)=-\delta_{x}$. The two first properties are straight forward from equation (5.22). When $x \neq y$ we have

$$
g(x, y)=\left\{\begin{array}{lll}
\int_{x}^{r} \frac{1}{\omega_{n} h^{n-1}(t)} d t & \text { if } & x \geq y \\
\int_{y}^{r} \frac{1}{\omega_{n} h^{n-1}(t)} d t & \text { if } & x \leq y .
\end{array}\right.
$$

Then, applying Proposition 5.9, we have

$$
\mathcal{L}_{x} g(x, y)=\left\{\begin{array}{ccc}
L_{0}\left(\int_{x}^{r} \frac{1}{\omega_{n} h^{n-1}(t)} d t\right)=0 & \text { if } & x \geq y \\
0 & \text { if } & y \leq x .
\end{array}\right.
$$

To prove condition $\mathcal{L}_{x} g(x)=,-\delta_{x}$, and using the definition of $\delta_{x}$, it is enough to prove that given $f \in \mathcal{F}$,

$$
f(x)=\int_{0}^{r}-\mathcal{L}_{x} g(x, y) f(y)=-\mathcal{L}_{x} \int_{0}^{r} g(x, y) f(y) d \mu(y), \quad \forall x .
$$

It is enough to show the identity (5.27) in $\Lambda$ then argue that (5.27) holds on the closure $\bar{\Lambda}$ by continuity. Let $f \in \Lambda$ and compute $\int_{0}^{r} g(x, y) f(y) d \mu(y)$.

$$
\begin{aligned}
\int_{0}^{r} g(x, y) f(y) d \mu(y)= & \int_{0}^{r}\left(\int_{x}^{r} \frac{\theta_{y}(t)}{h^{n-1}(t)} d t\right) f(y) h^{n-1}(y) d y \\
= & \int_{x}^{r} \frac{1}{h^{n-1}(t)}\left(\int_{0}^{t} \theta_{y}(t) f(y) h^{n-1}(y) d y\right. \\
& \left.+\int_{t}^{r} \theta_{y}(t) f(y) h^{n-1}(y) d y\right) d t \\
= & \int_{x}^{r} \frac{1}{h^{n-1}(t)}\left(\int_{0}^{t} f(y) h^{n-1}(y) d y\right) d t \\
= & T(f)(x) .
\end{aligned}
$$

Where $T$ is given in (2.10), viewed as an operator $T: C^{0}([0, r]) \rightarrow C^{0}([0, r])$. It is straight forward to show that $T(\Lambda) \subset \Lambda$. Thus, since $\mathcal{L}=L_{0}$ on $\Lambda$, to prove that $f(x)=-\mathcal{L}_{x} \int_{0}^{r} g(x, y) f(y) d \mu(y), \forall x$, it is enough to prove that $L_{0} \circ T=-i d_{\Lambda}$. This is done next.

$$
\mathcal{L} \circ T(u)=L_{0} \circ T(u)=\frac{\partial^{2} T(u)}{\partial t^{2}}+(n-1) \frac{h^{\prime}}{h}(t) \frac{\partial T(u)}{\partial t} .
$$


However,

$$
\begin{aligned}
\frac{\partial^{2} T(u)}{\partial t^{2}}(t) & =(n-1) \frac{h^{\prime}(t)}{h(t)} \frac{1}{h^{n-1}} \int_{0}^{t} h^{n-1}(s) u(s) d s-u(t) \\
(n-1) \frac{h^{\prime}(t)}{h(t)} \cdot \frac{\partial T(u)}{\partial t}(t) & =-(n-1) \frac{h^{\prime}(t)}{h(t)} \frac{1}{h^{n-1}(t)} \int_{0}^{t} h^{n-1}(s) u(s) d s .
\end{aligned}
$$

Then $\mathcal{L} \circ T(u)(x)=-u(x)$ and the proposition follows. To prove Theorem 5.4 we have from 5.21 that

$$
\begin{aligned}
\sum_{i=1}^{\infty} \frac{1}{\lambda_{i}^{\operatorname{rad}}\left(B_{h}(o, r)\right)} & =\int_{0}^{r} g(x, x) d \mu(x) \\
& =\int_{0}^{r}\left(\int_{x}^{r} \frac{d t}{h^{n-1}(t)}\right) h^{n-1}(x) d x \\
& =\int_{0}^{r} \frac{\int_{0}^{x} h^{n-1}(t) d t}{h^{n-1}(x)} d x \\
& =\int_{0}^{r} \frac{V(s)}{S(s)} d s,
\end{aligned}
$$

This proves identity (5.8). If $\mathbb{M}_{h}^{n}$ is stochastically incomplete, then its spectrum is discrete, say $\sigma\left(\mathbb{M}_{h}^{n}\right)=\left\{\lambda_{1}\left(\mathbb{M}_{h}^{n}\right)<\lambda_{2}\left(\mathbb{M}_{h}^{n}\right) \leq \cdots\right\}$. Taking the limits in (5.29) we obtain

$$
\lim _{r \rightarrow \infty} \sum_{i=1}^{\infty} \frac{1}{\lambda_{i}^{\operatorname{rad}}\left(B_{h}(o, r)\right)}=\int_{0}^{\infty} \frac{V(s)}{S(s)} d s<\infty .
$$

To prove identity (5.9) we recall that $\lambda_{i}^{\mathrm{rad}}\left(\mathbb{M}_{h}^{m}\right)=\lim _{r \rightarrow \infty} \lambda_{i}^{\mathrm{rad}}\left(B_{h}(o, r)\right)$. This proves that

$$
\sum_{i=1}^{\infty} \frac{1}{\lambda_{i}^{\mathrm{rad}}\left(\mathbb{M}_{h}^{n}\right)}=\int_{0}^{\infty} \frac{V(s)}{S(s)} d s<\infty
$$

Remark 5.11 In the proof of Theorem 5.4, we invoked Theorem 4.1 in order to show that the Green function of $L_{0}$ has finite trace. That could be also achieved by direct computation since the Green function of $L_{0}$ is given explicitly in Proposition 5.10 .

5.3.2. Proof of Theorem 5.6. The proof of Theorem 5.6 is similar to the proof of Theorem 5.4. The spectrum of $B_{h}(o, r)$, without repetitions, is the union of the $\nu_{l}$-spectrums $\sigma^{l}\left(B_{h}(o, r)\right), l=0,1, \ldots$

$$
\sigma\left(B_{h}(o, r)\right)=\cup_{l=0}^{\infty} \sigma^{l}\left(B_{h}(o, r)\right)=\left\{\lambda_{l, j}\right\}_{l=0, j=1}^{\infty, \infty},
$$

each $\lambda_{l, i}$ with multiplicity $\delta(l, m)$. The eigenvalues of the $l$-spectrum $\sigma^{l}\left(B_{h}(o, r)\right)$, $l \geq 1$, are the eigenvalues of the the operator

$$
L_{l}(T)(t)=T^{\prime \prime}(t)+(n-1) \frac{1}{t} T^{\prime}(t)-\frac{\nu_{l}}{t^{2}} T(t),
$$


$\nu_{l}=l(l+m-2)$, in the following Dirichlet eigenvalue problem on $[0, r]$.

$$
T^{\prime \prime}+(m-1) \frac{1}{t} T^{\prime}+\left(\lambda-\frac{\nu_{l}}{t^{2}}\right) T=0 \quad \text { in } \quad[0, r]
$$

with initial conditions $T(t) \sim c \cdot t^{l}$ as $t \rightarrow 0$ when $l=1,2 \ldots$ and $T(r)=0$. The procedure to show that the operator $L_{l}$ has a self-adjoint extension $\mathcal{L}_{l}$ and a Green function $g_{l}$ is similar to the procedure in the proof of Theorem 5.4. By Theorem 4.1 we have that

$$
\sum_{i=1}^{\infty} \frac{1}{\lambda_{l, i}\left(B_{h}(o, r)\right)}=\int_{0}^{r} g_{l}(x, x) d x
$$

We need to find the Green function $g_{l}$.

Proposition 5.12 The Green function $g_{l}(x, y)$ for the $\mathcal{L}_{l}$ operator on $M=[0, r]$ with density $d \mu(x)=\omega_{n} x^{n-1} d x$ boundary conditions

$$
u^{\prime}(0)=u(r)=0, \quad \text { with } u(x)=g_{l}(x, y) \text { for any } y \in(0, r)
$$

is given by

$$
g_{l}(x, y)= \begin{cases}\frac{x^{l} y^{\alpha}}{\beta \omega_{n} y^{n-1}}\left(\frac{1}{y^{\beta}}-\frac{1}{r^{\beta}}\right) & \text { if } \quad 0 \leq x<y \\ \frac{x^{l} y^{\alpha}}{\beta \omega_{n} y^{n-1}}\left(\frac{1}{x^{\beta}}-\frac{1}{r^{\beta}}\right) & \text { if } \quad y \leq x \leq r\end{cases}
$$

with $\alpha=l+n-1$ and $\beta=2 l+n-2$.

Observe first of all that

$$
u^{\prime}(0)=\left.\frac{\partial}{\partial x} g_{l}(x, y)\right|_{x=0}=0, \quad u(r)=g_{l}(r, y)=0, \quad \forall y \in[0, r]
$$

On the other hand

$$
\mathcal{L}_{l}(u(x))=u^{\prime \prime}(x)+(n-1) \frac{u^{\prime}(x)}{x}-\frac{l(l+n-2) u(x)}{x^{2}}=0
$$

because

$$
\mathcal{L}_{l}\left(x^{l}\right)=\mathcal{L}_{l}\left(x^{l-\beta}\right)=0 .
$$


For any function $f:[0, r] \rightarrow \mathbb{R}$, we have then

$$
\begin{aligned}
G_{l}(f)(x) & =\int_{0}^{r} g_{l}(x, y) f(y) d \mu(y) \\
& =\int_{0}^{x} g_{l}(x, y) f(y) d \mu(y)+\int_{x}^{r} g_{l}(x, y) f(y) d \mu(y) \\
& =\frac{x^{l}}{\beta}\left(\frac{1}{x^{\beta}}-\frac{1}{r^{\beta}}\right) \int_{0}^{x} y^{\alpha} f(y) d y+\frac{x^{l}}{\beta} \int_{x}^{r} y^{\alpha}\left(\frac{1}{y^{\beta}}-\frac{1}{r^{\beta}}\right) f(y) d y \\
& =\frac{x^{l-\beta}}{\beta} \int_{0}^{x} y^{\alpha} f(y) d y+\frac{x^{l}}{\beta} \int_{x}^{r} y^{\alpha-\beta} f(y) d y-\frac{x^{l}}{\beta r^{\beta}} \int_{0}^{r} y^{\alpha} f(y) d y .
\end{aligned}
$$

and

$$
\begin{aligned}
\mathcal{L}_{l}\left(G_{l}(f)(x)\right)= & \mathcal{L}_{l}\left(\frac{x^{l-\beta}}{\beta} \int_{0}^{x} y^{\alpha} f(y) d y\right)+\mathcal{L}_{l}\left(\frac{x^{l}}{\beta} \int_{x}^{r} y^{\alpha-\beta} f(y) d y\right) \\
& -\mathcal{L}_{l}\left(\frac{x^{l}}{\beta r^{\beta}}\right) \int_{0}^{r} y^{\alpha} f(y) d y \\
= & \mathcal{L}_{l}\left(\frac{x^{l-\beta}}{\beta}\right) \int_{0}^{x} y^{\alpha} f(y) d y+\mathcal{L}_{l}\left(\frac{x^{l}}{\beta}\right) \int_{x}^{r} y^{\alpha-\beta} f(y) d y \\
& -x^{l+\alpha-\beta-1} f(x) \\
= & -f(x),
\end{aligned}
$$

where we have applied $l+\alpha-\beta-1=0$. Therefore we conclude that,

$$
\mathcal{L}_{l} \circ G_{l}(f)(x)=-f(x),
$$

and $g_{l}$ is a Green function for our problem. Now

$$
\begin{aligned}
\sum_{i=1}^{\infty} \frac{1}{\lambda_{l, i}} & =\int_{0}^{r} g_{l}(x, x) d \mu(x)=\int_{0}^{r} \frac{x^{l+\alpha}}{\beta}\left(\frac{1}{x^{\beta}}-\frac{1}{r^{\beta}}\right) d x \\
& =\frac{r^{l+\alpha-\beta+1}}{(l+\alpha+1)(l+\alpha-\beta+1)} \\
& =\frac{r^{2}}{2(2 l+n)}=\left(\frac{1}{1+2 \frac{l}{n}}\right) \frac{r^{2}}{2 n} \\
& =\left(\frac{1}{1+2 \frac{l}{n}}\right) \max _{x \in B_{\mathbb{R}^{n}(r)}} E_{r}(x) .
\end{aligned}
$$


This proves (5.10). To prove (5.11) we proceed as follows.

$$
\begin{aligned}
\sum_{i=1}^{\infty} \frac{1}{\left(\lambda_{l, i}\right)^{2}} & =\int_{0}^{r} \int_{0}^{r} g_{l}(x, y) g_{l}(y, x) d \mu(y) d \mu(x) \\
& =\int_{0}^{r} \int_{0}^{r} \omega_{n}^{2} x^{n-1} y^{n-1} g_{l}(x, y) g_{l}(y, x) d y d x \\
& =\int_{0}^{r} \omega_{n}^{2} x^{n-1}\left(\int_{0}^{x} g_{l}(x, y) g(y, x) y^{n-1} d y+\int_{x}^{r} g(x, y) g_{l}(y, x) d y\right) d x \\
& =\frac{1}{\beta^{2}} \int_{0}^{r}\left(\int_{0}^{x} x^{l+\alpha} y^{l+\alpha}\left(\frac{1}{x^{\beta}}-\frac{1}{r^{\beta}}\right)^{2} d y+\int_{x}^{r} x^{l+\alpha} y^{l+\alpha}\left(\frac{1}{y^{\beta}}-\frac{1}{r^{\beta}}\right)^{2} d y\right) d x \\
& =\frac{r^{2(l+\alpha-\beta+1)}}{(\alpha+l+1)^{2}(\alpha+l-\beta+1)(2+2 \alpha-\beta+2 l)} \\
& =\frac{r^{4}}{2(2 l+n)^{2}(2+2 l+n)}
\end{aligned}
$$

\section{Spectrum of extrinsic balls of minimal submanifolds}

Let $\varphi: M \rightarrow \mathbb{R}^{n}$ be a proper and minimal immersion of a complete $m$-dimensional Riemannian manifold into $\mathbb{R}^{n}$. Let $\Omega_{r}=\varphi^{-1}\left(B_{h}(o, r)\right)$ be the extrinsic ball of radius $r$. It was proved in [6] and [12] that the first Dirichlet eigenvalue of $\Omega_{r}$ is bounded below as

$$
\lambda_{1}\left(\Omega_{r}\right) \geq \lambda_{1}\left(B_{h}(o, r)\right)=\frac{c_{m}^{2}}{r^{2}}
$$

where $c_{m}$ is a constant depending only on the dimension of $M$ and $\lambda_{1}\left(B_{h}(o, r)\right)$ is the first Dirichlet eigenvalue of the ball of radius $r$ in the Euclidean $m$-space $\mathbb{R}^{m}$. This inequality can be read as

$$
\frac{1}{\lambda_{1}^{2}\left(\Omega_{r}\right)} \leq C_{m} \cdot r^{4}
$$

where $C_{m}=1 / c_{m}^{4}$. By Theorem 4.1 we have that $\sum_{k=1}^{\infty} \frac{1}{\lambda_{k}^{2}\left(\Omega_{r}\right)}<\infty$ in dimensions $m=2,3$. For a totally geodesic $\mathbb{R}^{m} \subset \mathbb{R}^{n}$ with $m=2,3$ we have,

$$
\sum_{k=1}^{\infty} \frac{1}{\lambda_{k}^{2}\left(\Omega_{r}\right)}=D_{m} r^{4}
$$

where $D_{m}$ is a constant depending only on the dimension (see identity 5.12). For a non-totally geodesic minimal submanifold we will give lower and upper bounds for this series in terms of the volume $\operatorname{vol}\left(\Omega_{r}\right)$, of the radius $r$ and the dimension $m=2,3$. We have the following result. 
thmMark Theorem 6.1 Let $\varphi: M \rightarrow \mathbb{R}^{n}$ be a complete Riemannian m-manifold, properly and minimally immersed into the Euclidean $n$-space. Given $o \in M$, let $\Omega_{r}$ be the extrinsic r-ball with center o, namely,

$$
\Omega_{r}=\varphi^{-1}(B(\varphi(o), r)) .
$$

a. If $m=1,2,3$, then

$$
\left(\frac{A_{m} r^{m}}{\operatorname{vol}\left(\Omega_{r}\right)}\right) \cdot r^{4} \leq \sum_{k=1}^{\infty} \frac{1}{\lambda_{k}^{2}\left(\Omega_{r}\right)} \leq\left(\frac{\operatorname{vol}\left(\Omega_{r}\right)}{B_{m} r^{m}}\right)^{4 / m} \cdot r^{4},
$$

where $A_{m}=\frac{\omega_{m-1}}{m} \frac{1}{4 m^{2}}\left(1+\frac{m}{4+m}-\frac{2 m}{2+m}\right), B_{m}=\frac{2^{m} \pi^{\frac{m}{2}}}{e \cdot \zeta(4 / m)^{\frac{m}{4}}}$ and $\zeta(4 / m)=\sum_{k=1}^{\infty} \frac{1}{k^{4 / m}}$ are constants depending only on $m$.

b. If the second fundamental form $\alpha$ of the minimal immersion $\varphi: M \rightarrow \mathbb{R}^{n}$ has finite $L^{m}$-norm,

$$
\int_{M}\|\alpha\|^{m} d \mu<\infty
$$

then $M$ has finite number of ends $\left\{\operatorname{End}_{1}, \cdots, \operatorname{End}_{k}\right\}$ and

$$
\left(\frac{m A_{m}}{\omega_{m-1} \mathcal{E}}\right) \cdot r^{4} \leq \sum_{k=1}^{\infty} \frac{1}{\lambda_{k}^{2}\left(\Omega_{r}\right)} \leq\left(\frac{\omega_{m-1} \mathcal{E}}{m B_{m}}\right)^{4 / m} \cdot r^{4}
$$

where $\mathcal{E}$ is related with the finite number of ends of $M$ in the following way:

$$
\mathcal{E}=\left\{\begin{array}{ccc}
\sum_{i=1}^{k} I_{i} & \text { if } & m=2 \\
k & \text { if } & m=3,
\end{array}\right.
$$

where $I_{i}$ is the geometric index of the end $\operatorname{End}_{i}$.

6.0.1. Proof of Theorem 6.1. Let $\varphi: M \rightarrow N$ be a properly immersed $m$ submanifold $M$ into a Riemannian manifold $N$. Let $t_{N}(x)=\operatorname{dist}_{N}(p, x)$ be the distance in $N$ from a fixed point $p=\varphi(q) \in N$. Let $\Omega_{r}=\varphi^{-1}\left(B_{N}(p, r)\right)$ be an extrinsic ball that contains $q$, where $B_{N}(p, r)$ is the geodesic ball of $N$ with center at $p$ and radius $r<\min \{\operatorname{inj}(p), \pi / \sqrt{k}\}, k=\sup K_{N}$ and where $\pi / \sqrt{k}=\infty$ if $k \leq 0$.

Let $E_{r}(x)$ be the mean time of the first exit from $\Omega_{r}$ for a Brownian motion particle starting at $x \in \Omega_{r}$. A fundamental observation of Dynkin [17, vol.II, p.51] states that the function $E_{r}$ satisfies the Poisson equation

$$
\left\{\begin{aligned}
\triangle E_{r} & =-1 \text { in } \Omega_{r} \\
E_{r} & =0 \text { on } \partial \Omega_{r}
\end{aligned}\right.
$$


If $g^{\Omega_{r}}(x, y)=g(x, y)$ is the Green function of $\Omega_{r}$, with Dirichlet boundary data, then

$$
E_{r}(x)=\int_{\Omega_{r}} g(x, y) d \nu(y) .
$$

Applying Cauchy-Schwarz, assuming that $m=1,2,3$ and by Theorem 4.1, we obtain

$$
\begin{aligned}
\int_{\Omega_{r}} E_{r}^{2}(x) d \nu(x) & =\int_{\Omega_{r}}\left(\int_{\Omega_{r}} g(x, y) d \nu(y)\right)^{2} d \nu(x) \\
& \leq \operatorname{vol}\left(\Omega_{r}\right) \cdot \int_{\Omega_{r}} \int_{\Omega_{r}} g^{2}(x, y) d \nu(y) d \nu(x) \\
& =\operatorname{vol}\left(\Omega_{r}\right) \sum_{k=1}^{\infty} \frac{1}{\lambda_{k}\left(\Omega_{r}\right)^{2}}<\infty .
\end{aligned}
$$

Assume that $N=\mathbb{R}^{n}, p=0 \in \mathbb{R}^{n}$. Let $\widetilde{\mathbf{E}}_{r}: B^{m}(0, r) \rightarrow \mathbb{R}$ be the mean exit time of the first exit from the geodesic ball $B^{m}(0, r) \subset \mathbb{R}^{m}$. It is known that $\widetilde{\mathbf{E}}_{r}$ is radial, i.e. $\widetilde{\mathbf{E}}_{r}(y)=\widetilde{\mathbf{E}}_{r}(t(y)), t(y)=|y-o|, y \in \mathbb{R}^{m}$. Denote by $\widetilde{E}_{r}$ the transplant of $\widetilde{\mathbf{E}}_{r}$ to $B^{n}(0, r)$, i.e., the function $\widetilde{E}_{r}: B^{n}(0, r) \subset \mathbb{R}^{n} \rightarrow \mathbb{R}$ defined by $\widetilde{E}_{r}(z)=\widetilde{\mathbf{E}}_{r}(t(z))$. Consider the restriction of $\widetilde{E}_{r}(z)$ to the immersion $\varphi(M)$, i.e. $x \in \Omega_{r} \rightarrow \widetilde{E}_{r}(\varphi(x))$. In [31], Steen Markvorsen proved that if the immersion $\varphi: M \rightarrow \mathbb{R}^{m+1}$ is a minimal hypersurface then $E_{r}(x)=\widetilde{E}_{r}(\varphi(x))=\widetilde{\mathbf{E}}_{r}(t(\varphi(x)))$. Solving problem (6.3), we have

$$
\widetilde{\mathbf{E}}_{r}(t(x))=\int_{t(x)}^{r} \frac{\operatorname{vol}\left(B^{m}(0, \zeta)\right)}{\operatorname{vol}\left(\partial B^{m}(0, \zeta)\right)} d \zeta=\frac{1}{2 m}\left(r^{2}-t^{2}(x)\right),
$$

therefore, by inequality (6.4), we have

$$
\operatorname{vol}\left(\Omega_{r}\right) \sum_{k=1}^{\infty} \frac{1}{\lambda_{k}\left(\Omega_{r}\right)^{2}} \geq \frac{1}{4 m^{2}} \int_{\Omega_{r}}\left(r^{4}+t^{4}(x)-2 r^{2} t^{2}(x)\right) d \nu(x),
$$

where we identified $t(\varphi(x))=t(x)$. Applying co-area formula for the extrinsic distance function $t: M \rightarrow \mathbb{R}_{+}$we obtain

$$
\begin{aligned}
\int_{\Omega_{r}}\left(r^{4}+t^{4}(x)-2 r^{2} t^{2}(x)\right) d \nu(x) & =\int_{0}^{r}\left(\int_{\partial \Omega_{s}} \frac{r^{4}+t^{4}(x)-2 r^{2} t^{2}(x)}{|\nabla t|} d A\right) d s \\
& =\int_{0}^{r}\left(r^{4}+s^{4}-2 r^{2} s^{2}\right)\left(\int_{\partial \Omega_{s}} \frac{1}{|\nabla t|} d A\right) d s \\
& \geq \int_{0}^{r}\left(r^{4}+s^{4}-2 r^{2} s^{2}\right) \operatorname{vol}\left(\partial \Omega_{s}\right) d s .
\end{aligned}
$$

On the other hand, we have that $\operatorname{vol}\left(\partial \Omega_{s}\right) \geq \omega_{m-1} s^{m-1}$, see [34]. Then

$$
\begin{aligned}
\int_{\Omega_{r}}\left(r^{4}+t^{4}(x)-2 r^{2} t^{2}(x)\right) d V(x) & \geq \omega_{m-1} \int_{0}^{r}\left(r^{4}+s^{4}-2 r^{2} s^{2}\right) s^{m-1} d s \\
& =\omega_{m-1} r^{4+m}\left(\frac{1}{m}+\frac{1}{4+m}-\frac{2}{2+m}\right) .
\end{aligned}
$$


In order to simplify the notation let us denote by $A_{m}:=\frac{\omega_{m-1}}{m} \cdot \frac{1}{4 m^{2}}\left(1+\frac{m}{4+m}-\frac{2 m}{2+m}\right)$. Hence,

$$
\operatorname{vol}\left(\Omega_{r}\right) \cdot \sum_{k=1}^{\infty} \frac{1}{\lambda_{k}\left(\Omega_{r}\right)^{2}} \geq r^{4} \cdot A_{m} \cdot r^{m}
$$

That proves the lower bound of item $a$. of Theorem 6.1. To prove the upper bound recall that Cheng, Li and Yau proved in [12] that

$$
\lambda_{k}\left(\Omega_{r}\right) \geq 4 \pi\left(\frac{k}{e}\right)^{2 / m} \frac{1}{\operatorname{vol}\left(\Omega_{r}\right)^{2 / m}} .
$$

Therefore,

$$
\sum_{k=1}^{\infty} \frac{1}{\lambda_{k}\left(\Omega_{r}\right)^{2}} \leq \frac{e^{4 / m}}{16 \pi^{2}} \operatorname{vol}\left(\Omega_{r}\right)^{4 / m} \sum_{k=1}^{\infty} \frac{1}{k^{4 / m}}
$$

$$
=\frac{e^{4 / m}}{16 \pi^{2}} \operatorname{vol}\left(\Omega_{r}\right)^{4 / m} \zeta(4 / m)
$$

Observe that $\zeta(2)=\pi^{2} / 6$. Putting together inequalities (6.7) and (6.9) we obtain

$$
\left(\frac{A_{m} r^{m}}{\operatorname{vol}\left(\Omega_{r}\right)}\right) \cdot r^{4} \leq \sum_{k=1}^{\infty} \frac{1}{\lambda_{k}^{2}\left(\Omega_{r}\right)} \leq\left(\frac{\operatorname{vol}\left(\Omega_{r}\right)}{B_{m} r^{m}}\right)^{4 / m} \cdot r^{4} .
$$

Here $B_{m}=\frac{2^{m} \pi^{\frac{m}{2}}}{e \cdot \zeta(4 / m)^{\frac{m}{4}}}$ and $\zeta(4 / m)=\sum_{k=1}^{\infty} \frac{1}{k^{4 / m}}$. In order to obtain inequality of the item $b$. we observe that by the monotonicity formula the function

$$
r \rightarrow \frac{\operatorname{vol}\left(\Omega_{r}\right)}{V_{m} r^{m}}, \quad V_{m}:=\frac{\omega_{m-1}}{m} .
$$

is increasing, (see [33, 34]). Moreover by the classical results of Jorge-Meeks in [27], see also $[2,36]$,

$$
\lim _{r \rightarrow \infty} \frac{\operatorname{vol}\left(\Omega_{r}\right)}{V_{m} r^{m}}=\mathcal{E}
$$

Therefore

$$
\operatorname{vol}\left(\Omega_{r}\right) \leq V_{m} \mathcal{E} r^{m}
$$

and the theorem follows taking in consideration that

$$
\mathcal{E}=\left\{\begin{array}{rrr}
\sum_{i=1}^{k} I_{i} & \text { if } & m=2 \\
k & \text { if } & m=3
\end{array}\right.
$$

where $I_{i}$ is the geometric index of the end $E_{i}$, see details in [27]. 


\section{References}

Ahlfors

Anderson

berger

berger-gauduchon

barroso-bessa

bessa-montenegro-2007

bessa-montenegro-2008

bessa-montenegro-2009

bessa-pigola-setti

$\mathrm{bmr}$

chavel

CLY

C-L

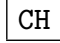

davies 2

davies

dynkin

Fischer

[1] V. L. Ahlfors: Sur le type d'une surface de Riemann. C. R. Acad. Sci. Paris 201 (1935), 30-32, Zbl 0011.40701.

[2] M. Anderson: The compactification of a minimal submanifold in Euclidean space by the Gauss map. Preprint IEHS, (1986).

[3] M. Berger: A panoramic view of Riemannian Geometry. Springer-Verlag, Berlin, 2003, xxiv+824 pp. ISBN:3-540-65317-1

[4] M. Berger, P. Gauduchon and E. Mazet: Le spectre d'une variété Riemannienne. Lecture Notes in Mathematics 194, Springer Berlin Heidelberg, 1971, ISBN: 978-3-540-05437-5.

[5] C. Barroso and G. P. Bessa: Lower bounds for the first eigenvalue of geodesic ball of spherically symmetric manifolds. Int. J. Appl. Math. Stat. 6 (2006), no.6 $82-86$.

[6] G. P. Bessa and J. F. Montenegro: An extension of Barta's theorem and geometric applications. Ann. Global Anal. Geom. 31 (2007), no.4 345-362.

[7] G. P. Bessa and J. F. Montenegro: On Cheng's eigenvalue comparison theorem. Math. Proc. Cambridge Philos. Soc 144 (2008), no.3 673-682.

[8] G. P. Bessa And J. F. Montenegro: Mean time exit and isoperimetric inequalities for minimal submanifolds of $N \times \mathbb{R}$. Bull. London. Math. Soc. 41 (2009), 242-252.

[9] G. P. Bessa, S. Pigola and A. G. Setti: Spectral and stochastic properties of the $f$-Laplacian, solutions of PDE's at infinity and geometric applications. Rev. Mat. Iberoam. 29 (2013), no.2 579-610.

[10] B. Bianchini, L. Mari, M. Rigoli: On some aspect of oscillation theory and geometry.. Mem. Amer. Math. Soc. 225 (2013),no. 1056, vi+195 pp. ISBN: 978-08218-8799-8.

[11] I. Chavel: Eigenvalues in Riemannian Geometry. Including a chapter by Burton Randol. With an appendix by Jozef Dodziuk. Pure and Applied Mathematics 115. Academic Press, Inc., Orlando, FL, 1984, xiv+363 pp. ISBN: 0-12-170640-0.

[12] S. Y. Cheng, P. Li And S. T. Yau: Heat equations on minimal submanifolds and their applications. Amer. J. Math. 106 (1984), no. 5 1033-1065.

[13] E. A. Coddington And N. Levison: Theory of ordinary differential equations. McGraw-Hill, 1955, 429 pp. ISBN: 0898747554

[14] R. Courant and D. Hilbert: Methods of Mathematical Physics, Vol.1,First English Edition. Interscience Publishers, Inc. New York, 1953.

[15] E. B. DAviEs: Heat kernel and Spectral Theory. Cambridge Tracts in Mathematics 92. Cambridge University Press, 1989. ISBN:978-0-521-36136-1.

[16] E. B. Davies: Spectral theory and differential operators. Cambridge Studies in Advanced Mathematics 42. Cambridge University Press, 1995. ISBN:0-521-47250-4.

[17] E. B. Dynkin: Markov Processes. Vol.I, II. Translated with the authorization and assistence of the author by J. Fabius, V. Greenberg, A. Maitra, G. Majone. Die Grundlehren der Mathematischen Wissenschaften, Bände 121, vol; 122, Academic Press Inc. Publishers, New York, 1965.

[18] E. Fisher: Sur la convergence en moyenne. C.R. Acad. Sci. Paris 144 (1907), 10221023 \& 1148-1150. 
Grigoryan89 [19] A. GRIGOR'YAN: Stochastically incomplete manifolds and summable functions. Math. USSR Izvestya 33 (1989), no.2 425-432.

Grigoryan99 [20] A. GRIGOR'YAN: Analytic and geometric background of recurrence and nonexplosion of the Brownian motion on Riemannian manifolds. Bull. Amer. Math. Soc. (N.S.) 36 (1999), 135-249.

grigoryan2006 [21] A. GRIGOR'YAN: Heat kernels on weighted manifolds and applications. The ubiquitious heat kernel. Contemp. Math. 398 (2006), 93-191.

grigoryan-book [22] A. Grigor'YAN: Heat $K$ and Analysis on Manifolds. S. T. Yau, series editor, AMS/IP Studies in Advanced Mathematics 47. American Mathematical Society, Providence, RI. International Press Boston, MA, 2009, xviii+482 pp. ISBN: 978-082118-4935-4.

Gri09 [23] A. GRIGOR'YAn, JiaXn Hu AND KA-Sing LAU: Heat kernels on metric spaces with doubling measure. In Fractal geometry and stochastics IV, Prog. Probab.., 61, 3-44. Birkhäuser Verlag, Basel, 2009.

gruter-widman [24] M. Grüter and KJell-Ove Widman: The Green function for uniformly elliptic equations. Manuscripta Math. 37 (1982), no.3 303-342.

HMP

[25] A. Hurtado, S. Markvorsen and V. Palmer: Estimates of the first Dirichlet eigenvalue from exit time moment spectra.. Math. Ann. 365 (2016), no. 3-4 16031632.

[26] K. IChinara: Curvature, geodesics and the Brownian motion on a Riemannian manifolds I. Recurrence properties. Nagoya Math. J. 87 (1982), 101-114.

[27] L. P. Jorge And W. Meeks III: The topology of complete minimal surfaces of finite total curvature. Proc. London Math. Soc 93 (2006), no. 3 253-272.

[28] P. MacDonald: Isoperimetric conditions, Poisson problems and diffusion in Riemannian manifolds. Potential Anal. 16 (2002), 115-138.

[29] P. MacDonald and R. Meyers: Dirichlet spectrum and heat content. J. Funct. Anal. 200 (2002), no. 1 150-159.

Mc2 [30] P. MACDonald: Exit times, moment problems and comparison theorems. Potential Anal. 38 (2013), 1365-1372.

Mar [31] S. MARKVORSEn: On the heat kernel comparison theorems for minimal submanifolds. Proc. Amer. Math. Soc 97 (1986), 479-482.

[32] S. Markvorsen and V. Palmer: Torsion rigidity of minimal submanifold. Proc. London Math. Soc 93 (2006), no. 3 253-272.

[33] W. H. Meeks III And J. PÉREz: A survey on classical minimal surface theory. University Lecture Series, 60, American Mathematical Society, Providence RI, 2012.

Palmer

perelman

[34] V. PALmer: Isoperimetric inequalities for extrinsic balls in minimal submanifolds and their applications. J. London Math. Soc 60 (1999), no. 2 607-616.

[35] G. Perelman: A complete Riemannian manifold of positive Ricci curvature with Euclidean volume growth and non-unique asymptotic cone. Comparison geometry., 165-166. Math. Sci. res. Inst, Publ. 30, Cambridge Univ. Press, Cambridge (1997).

[36] Chen Quing: On the volume growth and the topology of minimal submanifolds of an Euclidean space. J. Math. Sci. Univ. Tokyo 2 (1995), 657-669.

[37] F. Riesz: Sur les systmes orthogonaux de fonctions. C.R. Acad. Sci. Paris 144 (1907), 615-619.

[38] S. SAto: Barta's inequality and the first eigenvalue of a cap domain of a 2 -sphere. Math. Z. 181 (1982), 313-318. 
Received ??

G. P. Bessa: Department of Mathematics, Universidade Federal do Ceará, 60440900 Fortaleza, Brazil.

E-mail: bessa@mat.ufc.br

V. Gimeno: Department of Mathematics, Universitat Jaume I-IMAC, E-12071, Castelló, Spain.

E-mail: gimenov@mat.uji.es

L. Jorge: Department of Mathematics, Universidade Federal do Ceará, 60440-900 Fortaleza, Brazil.

E-mail: ljorge@mat.ufc.br

The first and third authors were partially supported by CNPq-grants \# 301581/2013-4 Projeto Universal-CNPq \# 445993/2014-6 and Pronex-CNPq-FUNCAP, Brazil. The second author was partially supported by the Research Program of University Jaume I Project UJI-B2016-07 and DGI -MINECO Grant (FEDER) MTM2013-48371-C2-2-P, Spain. 